\title{
Reduction of spectral interferences and noise effects in laser ablation molecular isotopic spectrometry with partial-least square regression - a computer simulation study
}

Xianglei Mao, George C.-Y. Chan", Vassilia Zorba, Richard E. Russo Lawrence Berkeley National Laboratory, Berkeley, CA 94720, USA

*corresponding author, email: gcchan@lbl.gov

(C) 2016. This manuscript version is made available under the Elsevier user license http://www.elsevier.com/open-access/userlicense/1.0/ 


\section{Abstract}

The fundamental analytical accuracies and precisions attainable by laser ablation molecular isotopic spectrometry (LAMIS), with emphasis on the impacts from spectral interferences and measurement noise, were investigated by means of computer simulation. The study focused on the analysis of a minor isotope at sub- to single-percentage abundance level. With a natural abundance about $1.1 \%$ for ${ }^{13} \mathrm{C}$, the $\mathrm{C}_{2}$ Swan band $\left(d^{3} \Pi_{\mathrm{g}}-a^{3} \Pi_{\mathrm{u}}\right)$ with $\Delta v=+1$ was selected as a representative system. The characteristics (e.g., noise amplitude and distribution, signal strength, and signal-to-background ratio) of the simulated spectra were experimentally characterized. Partial least square (PLS) regression was used to extract isotopic information from the simulated molecular spectra. In the absence of any spectral interference and with the use of a calibration set consisting of eleven isotopic standards, the theoretical accuracies and precisions with signal accumulation from 100 laser shots are about $0.002 \%$ and $0.001 \%$, respectively, in absolute percentage abundance of ${ }^{13} \mathrm{C}$. The theoretical analytical accuracies slightly degrade, but are adequate for many applications, to $0.004 \%$ and $0.008 \%$ respectively, for calibrations involving only three and two isotopic standards. It was found that PLS regression is not only immune to both source-flicker and photon-shot noise, but is also effective in differentiating the spectral patterns from the analyte against those from spectral interferences. The influences of spectral interference from single or multiple atomic emission lines were simulated, and new ways to minimize their impacts were formulated and demonstrated. It was found that the wavelength range selected for the computation of the normalization factor should not contain any spectralinterfering peak, and a properly chosen wavelength range increases the tolerance of spectral interference by at least one order of magnitude. With matrix-matched calibration standards, the

precisions (expressed as RSDs of the determined ${ }^{13} \mathrm{C}$ isotopic abundances) degrade from $\sim 1 \%$ in 
the absence of spectral interference, to $\sim 3 \%, 10 \%$ and $20 \%$ with spectral-interfering peaks that are $10 \times, 100 \times$ and $1000 \times$, respectively, stronger than the molecular bandhead of the analyte. The study concluded that PLS regression is a powerful and indispensable tool for extraction of isotopic information from LAMIS spectra.

Keywords: Isotopic analysis; Laser ablation molecular isotopic spectrometry; Partial least square; Spectral interference; $\mathrm{C}_{2}$ Swan band 


\section{Introduction}

Laser ablation molecular isotopic spectrometry (LAMIS) is newly developed all-optical technique for isotopic analysis at ambient atmospheric pressure [1-3]. The implementation of LAMIS is similar to the well-known laser induced breakdown spectroscopy (LIBS) technology, in which a laser beam is focused onto the surface of a sample, thereby initiating a plasma above the sample surface. As time evolves, the plasma expands and the dominant species in the plasma shift from ions, to atoms, molecules, and finally fine particles. LIBS measurement involves atomic emission from the ions and neutral-atoms whereas LAMIS measures the molecular spectra from those radicals that are present when the plasma cools. Because the vibrational and rotational motions of a molecule heavily depend on its reduced mass, which in turn depends on the molecular isotopologue, molecular spectra may exhibit up to two to three orders of magnitude increase in isotopic shifts compared to electronic transitions in atomic spectra [1-3]. Consequently, despite the various line broadening mechanisms operating inside the atmospheric pressure laser-induced plasma, isotopic shifts in molecular spectra are readily measurable with an optical spectrometer of relatively low spectral resolution. In addition, similar to LIBS, the alloptical nature of LAMIS offers the capability of remote analysis in any atmosphere without the need for vacuum equipment. For instance, we recently demonstrated the analysis of zirconium isotopes using a femtosecond laser-induced filament from a sample at a distance of $7.8 \mathrm{~m}-\mathrm{a}$ technique coined $\mathrm{F}^{2}$-LAMIS (Femtosecond Filament LAMIS) [4].

Despite the fact that utilizing molecular emission for the determination of isotopic abundance ratio in a sample is a concept with a long history $[5,6]$, it has been relatively recently that isotope ratios were measured through molecular spectra with laser as the excitation source. The first 
study utilizing the laser induced plasma for isotopic analysis based on molecular spectra was reported by Niki et al. [7] in 1998. Under operation at a reduced pressure of $4 \mathrm{hPa}$, they demonstrated the marked differences in the BO $A^{2} \Pi-X^{2} \Sigma(0,2)$ molecular bands emitted from the two isotopologues, ${ }^{10} \mathrm{BO}$ and ${ }^{11} \mathrm{BO}$, and reported that the isotopic compositions of the two isotopologues could be measured from their relative peak heights [7]. The claimed detectability was only $5 \%$ (i.e., able to detect $>5 \%$ difference in isotopic abundance) from the natural abundance of boron [7]. In our first demonstration studies of LAMIS under atmospheric pressure, we used a nanosecond-pulsed laser for isotopic analysis of boron [2, 3], also utilizing the BO $A^{2} \Pi-X^{2} \Sigma^{+}$band. The reported precisions, expressed as standard deviations of the percentage compositions of ${ }^{11} \mathrm{~B}$, ranged from 0.9 to $3.7 \%$ for a boron-nitride solid sample at natural isotopic abundance. Jovanovic and co-workers $[8,9]$ also performed LAMIS of boron but with a femtosecond laser, and reported precisions $\sim 1.9$ to $3.0 \%$ on the measured isotopic composition. Sarkar et al. [10] advanced the analytical capabilities of LAMIS for boron isotopic analysis through a detailed characterization of the factors limiting the analytical accuracy and precision, and found that a critical factor is the data-pretreatment procedure to remove shot-toshot spectral variations prior to partial least square (PLS) regression. With several optimization procedures, Sarkar et al. [10] reported an improvement in the precision of determined boron isotopic composition to $2 \%$ RSD with a 1000-laser shots measurement. Hydrogen is another element with its isotopic ratio quantitatively determined by LAMIS [11]. Through the measurement and analysis of the $\mathrm{OH}$ and $\mathrm{OD} A^{2} \Sigma^{+}-X^{2} \Pi$ band, Sarkar et al. [11] reported accuracies from $0.5 \%$ to $1.5 \%$ and precision from $15 \%$ to $18 \%$ RSD on the isotopic abundances of deuterium. In all the above examples, the minor isotopes were present at relatively high abundances. The boron figures quoted above were all from boron samples with natural isotopic 
abundance $[2,3,7-10]$ (the minor isotope, ${ }^{10} \mathrm{~B}$ is $\sim 19.9 \%$ [12]) whereas the atom percentages of ${ }^{2} \mathrm{H}$ in the OH/OD LAMIS study [11] ranged from $6 \%$ to $97 \%$. There are many isotopes with natural abundances around or less than $1 \%$ (e.g. ${ }^{13} \mathrm{C} \sim 1.1 \%,{ }^{15} \mathrm{~N} \sim 0.4 \%,{ }^{18} \mathrm{O} \sim 0.2 \%,{ }^{235} \mathrm{U} \sim 0.7 \%$ [12]). One objective of the present study is to understand the fundamental limits of analytical accuracy and precision attainable by LAMIS when the minor isotopes are at abundances $\sim 1 \%$.

As many samples contain other co-existing elements besides the element(s) of interest, spectral interferences could potentially limit the accuracy and precision of LAMIS. Brown et al. [13] recently showed that, for isotopic analysis of $\mathrm{B}$ using the $\mathrm{BO}$ molecular emission in a $\mathrm{LaBO}_{3}$ sample, La atomic lines strongly overlapped the BO molecular band and thus compromised the analysis. Even for a highly purified sample that contains only the analyte, because atomic emission sometimes overlaps with molecular emission in both the temporal and wavelength domains, the ionic or neutral atomic emission from the analyte itself is a potential source of spectral interference in LAMIS. For instance, in our recent $\mathrm{Zr}$ isotopic analysis with LAMIS [14] on a pure zirconium metallic plate, $\mathrm{Zr}$ I emission lines overlap with the $\mathrm{ZrO}$ band under measurement. As is true with any other analytical technique, the presence of such spectral interferences, without subsequent correction by the analyst, can degrade the analytical performance of LAMIS. In order to further advance the LAMIS technique, it is of prime importance to understand the influence of spectral interference on the analytical accuracy and precision, especially when the minor isotope is present at sub- or single-percentage concentration.

One common approach to extract isotopic abundance information from molecular emission spectra is through multivariate calibration with partial least square (PLS) regression $[15,16]$. 
The ideal approach to investigate the precision and accuracy of the LAMIS technique with PLS calibration is through experimental means - such as physically prepare a set of calibration standards and reference samples with known isotopic abundances, experimentally acquire the spectra with subsequent PLS processing, and compare the predicted results with the reference values. However, such an all-experimental approach is time consuming and it is sometimes difficult to precisely control the variable under investigation. For example, as will be discussed in detail later in this paper, one factor that we explored is the effect of the center wavelength of a spectral-interfering line with respect to the positions of the molecular band features (i.e., band head or tail). As it is practically infeasible to prepare a sample with spectral interference at any desirable wavelength, computer simulation is a more viable approach for such a study. In addition, computer simulation allows us to identify the dominant factors influencing the analytical performance of LAMIS, and hence provides valuable guidelines for optimization prior to a measurement.

In this study, computer simulation was employed to estimate the analytical performance of LAMIS with PLS calibration. The focus of this work was to understand the limiting factors for LAMIS when the abundance of the minor isotope is at sub- or single-percentage levels, with emphasis on spectral interference from other co-existing elements in the sample. In addition to understanding how different factors affect the analytical performance of LAMIS, new ways to minimize their impacts were also formulated and demonstrated. The $\mathrm{C}_{2}$ Swan band $\left(d^{3} \Pi_{\mathrm{g}}-\right.$ $\left.a^{3} \Pi_{\mathrm{u}}\right)$ with $\Delta v=+1$ was selected as the representative system, as the natural abundance of ${ }^{13} \mathrm{C}$ is about $1.1 \%$. Further, the $\mathrm{C}_{2}$ Swan band is one of the strongest molecular bands present when an organic material is ablated [17, 18], and therefore, has been utilized for isotopic analysis of carbon [19-21]. Although the spectra of both the standards and the samples are synthetic in 
nature, their characteristics (e.g., noise amplitude and distribution, signal strength, and signal-tobackground ratio) were all experimentally characterized.

The reported accuracy and precision from computer simulation represent the theoretical limit of the analytical figures of merit of LAMIS. Even though the theoretical limit is likely an optimistic value, such knowledge is still valuable. First, as LAMIS is a new technique for isotopic analysis, the theoretical value serves as a guide on its analytical figures of merit under an idealized situation. Although the required precision for a particular analysis depends on the specific application, many isotopic analyses would require precisions at or better than per-mil level to match the similar levels of natural variations in isotopic abundance of many elements. If the theoretical limit predicted for a particular molecular isotopic system is very close or short from the requirement, it directly suggests that LAMIS is not a suitable candidate for that particular application. Second, if large gaps are found between experimental analytical figure of merit and the theoretical value predicted under flawless situation, it prompts the researcher to look for, and accordingly remediate, the source of error (e.g., quality of PLS calibration standards, shot-to-shot variations in plasma temperature, possibility of the presence of selfabsorption).

\section{Experimental}

\subsection{Sample and experimental setup}

The experimental setup was similar to previously described [21]. Briefly, a Nd:YAG laser (Brilliant Eazy, Quantel) operating at $1064 \mathrm{~nm}$, with pulse duration of $5 \mathrm{~ns}$ and energy of 
$115 \mathrm{~mJ}$, was focused onto the sample surface with a fused-silica lens (focal length $=5 \mathrm{~cm}$ ). The sample was a graphite plate with thickness of $1.3 \mathrm{~cm}$ (MFCD00144065, Alfa Aesar). The measured laser spot size on the sample was approximately $2.5 \mathrm{~mm}$. Plasma emission was collected through a $50 \mathrm{~mm}$-diameter lens and imaged with a magnification ratio of 1:1 onto the entrance slit of a Czerny-Turner spectrometer (IsoPlane SCT-320, Princeton Instruments). An intensified charge-coupled device (ICCD) (Princeton Instruments) detector was mounted at the exit focal plane of the spectrometer. The ICCD was operated with a gain setting of 200 (out of 255). A grating with groove density of 2400 per $\mathrm{mm}$ was used. The center wavelength of the spectrometer was set at $472 \mathrm{~nm}$. With these settings, the wavelength coverage on the ICCD detector spans $12 \mathrm{~nm}$, from 466 to $478 \mathrm{~nm}$. The measured spectral resolution (expressed as full width at half maximum, FWHM) was about $60 \mathrm{pm}$. Operating parameters of the ICCD were set at $1 \mu$ s delay time with a gate width of $40 \mu$ s. The ICCD was operated in two-dimensional imaging mode. The 2D-image was reduced to a $1 \mathrm{D}$-spectrum by summation of all the signals acquired along the spectrometer-slit axis during data processing.

\subsection{Characterization of measurement noise}

Source flicker and photon shot are two identified major noise sources in emission spectra measured from a laser induced plasma [22]. Source flicker noise is correlated and is related to the fluctuations inside the laser induced plasma with standard deviations directly proportional to the signals, whereas photon shot noise is uncorrelated and is related to the fundamental random nature of photon arrival onto the detector [22]. In addition, detector read noise, although minor, was also incorporated into the simulated molecular spectra. The noise levels from these three sources were experimentally determined in the same fashion as described in our previous study 
[23]. Specifically, the photon transfer curve of the ICCD detector was used to determine both the detector-read and photon-shot noise [24]. The source-flicker noise level was characterized from experimentally acquired LAMIS measurements of the $\mathrm{C}_{2}$ Swan band. The experimental condition and plasma-light collection efficiency were first optimized for maximum $\mathrm{C}_{2}$ emission. The strong $\mathrm{C}_{2}$ emission helps to warrant that flicker is the dominant noise source and the contribution from shot noise is comparatively minor. After optimization, a total of 600 spectra, each acquired with a single-shot laser, were recorded to determine the stability of $\mathrm{C}_{2}$ emission. The stage with the graphite sample was translated at a speed of $0.1 \mathrm{~mm} / \mathrm{s}$ during spectra acquisition with the laser firing at a repetition rate of $1 \mathrm{~Hz}$. Figure 1 depicts the distribution of the peak heights of the ${ }^{12} \mathrm{C}^{12} \mathrm{C}(1-0)$ bandhead at $473.73 \mathrm{~nm}$. The $\mathrm{C}_{2}$ emission fluctuations were well represented by a Gaussian distribution with a mean signal of $8.3 \times 10^{6}$ and a standard deviation of $2.0 \times 10^{6}$ in units of ICCD counts. The relative standard deviation (RSD) of the $\mathrm{C}_{2}$ Swan (1-0) band with single-shot laser was $24 \%$. The experimentally measured detector read, photon shot and source flicker noise levels were independently added to all simulated spectra. Photon-shot noise was assumed to behave according to Poisson statistics whereas both sourceflicker and detector-read noise were modelled with Gaussian distributions. The characterized photon-shot noise $\left(N_{\text {photon-shot }}\right)$ followed the relationship: $N_{\text {photon-shot }}=6.78(\text { Signal })^{0.498}$, where Signal is in unit of ICCD counts.

\subsection{Computer simulation}

As mentioned in the introduction, the $\mathrm{C}_{2} d^{3} \Pi_{\mathrm{g}}-a^{3} \Pi_{\mathrm{u}}$ (Swan) band was selected as a representative system for the simulation of the analytical performance of LAMIS. Apart from the reasons already stated, the choice of the $\mathrm{C}_{2}$ Swan band is also associated with the fact that its 
spectroscopic constants have been reported by various research groups [25, 26]. Also, extensive line lists for its rovibronic transitions are readily available for all its isotopologues [26-28]. In this study, the spectral positions of the rovibronic lines, their energy levels, statistical weights and transition probabilities for the triplet branches of ${ }^{12} \mathrm{C}_{2}$ and ${ }^{13} \mathrm{C}^{12} \mathrm{C}$ bands were adapted from Brooke et al. [27] and Kurucz [28], respectively. For ${ }^{12} \mathrm{C}_{2}$, Brooke et al. [27] listed both the observed and calculated line positions for most (but not all) transitions. In our simulation, the experimentally observed line positions were used if listed; otherwise, the calculated line positions were used. For the simulation of the ${ }^{13} \mathrm{C}^{12} \mathrm{C}$ spectra, the energy level of the lower electronic state $-a^{3} \Pi_{\mathrm{u}}-$ was shifted downward by $16.3 \mathrm{~cm}^{-1}$. This downward shift was found to be necessary to match the line positions listed in the Kurucz database [28] to our experimental line positions, and is in accordance with the molecular constants published by Amiot [29]. After the correction, although a small percentage of the simulated ${ }^{13} \mathrm{C}^{12} \mathrm{C}$ line positions still exhibited slight mismatches in emission wavelengths compared to our experimental acquired ${ }^{13} \mathrm{C}^{12} \mathrm{C}$ spectra, most of them agreed to within 1 detector pixel. For the purpose of the present study, the corrected Kurucz database [28] was adequate for spectra simulation.

Figure 2a shows the simulated $\mathrm{C}_{2}$ Swan band - the individual ${ }^{12} \mathrm{C}^{12} \mathrm{C}$ and ${ }^{13} \mathrm{C}^{12} \mathrm{C}$ components, and their sum after background continuum and noise additions. The $\mathrm{C}_{2}$ Swan band with $\Delta v=+1$ is an appropriate choice for isotopic analysis of ${ }^{13} \mathrm{C}$ because the line positions from the ${ }^{13} \mathrm{C}^{12} \mathrm{C}$ isotopologue is red shifted from the more abundant ${ }^{12} \mathrm{C}^{12} \mathrm{C}$. As the bandheads of the $\mathrm{C}_{2} d^{3} \Pi_{\mathrm{g}}-$ $a^{3} \Pi_{\mathrm{u}}$ system degrade to blue, the red-shifted ${ }^{13} \mathrm{C}^{12} \mathrm{C}(1-0)$ bandhead appears in front of and not buried inside the decaying part of the dominant ${ }^{12} \mathrm{C}_{2}$ bandhead. However, other rovibronic structures from the Swan band, in particular those from $0-0,1-1$ and 9-8 [21], of the dominant 
${ }^{12} \mathrm{C}_{2}$ isotopologue emit in close wavelength proximity to the bandhead of ${ }^{13} \mathrm{C}^{12} \mathrm{C} d{ }^{3} \Pi_{\mathrm{g}}-a^{3} \Pi_{\mathrm{u}}$ $(1-0)$ at $474.48 \mathrm{~nm}$.

The line positions for the (9-8) Swan band, as well as those (0-0) and (1-1) bands with rotational numbers $<100$, are well documented by Brooke et al. [27]. For those $\mathrm{R}(0-0)$ and $\mathrm{R}(1-1)$ Swan band with high $(\geq 100)$ rotational numbers that emit between 473.8 to $476.5 \mathrm{~nm}$, their experimental line positions were reported only recently [21]. Accordingly, in our simulation, line positions for the high rotational $\mathrm{R}(0-0)$ and $\mathrm{R}(1-1)$ Swan band for ${ }^{12} \mathrm{C}_{2}$ were directly taken from Bol'shakov et al. [21] whereas the rotational energy levels and transition probabilities for those high rotational lines were extrapolated from the data published by Brooke et al. [27].

Lorentzian line profiles were used for the generation of the simulated emission spectra. Unless otherwise specified, a line width of $60 \mathrm{pm}$, which was experimentally determined, was used for all emission lines. The separation between adjacent spectral-data points was $11.5 \mathrm{pm}$, and corresponded to the wavelength coverage of one ICCD pixel. The spectral range under simulation was from 466 to $478 \mathrm{~nm}$, which again matched with our experimental spectra. As the experimentally determined rotational temperature of $C_{2}$ (with a 1- $\mu$ s gate delay and a $40-\mu$ s gate width) was in the range of $5000 \mathrm{~K}$, all molecular spectra were simulated with a temperature of $5000 \mathrm{~K}$.

The ${ }^{12} \mathrm{C}_{2}(1-0)$ bandhead at $473.73 \mathrm{~nm}$ was used as a reference to define the emission intensity of the simulated $\mathrm{C}_{2}$ Swan band. Random Gaussian noise with a magnitude defined by the flicker noise level (i.e., 24\%, cf. Figure 1) was generated and then added to the mean measured emission 
of the $\mathrm{C}_{2}(1-0)$ Swan bandhead. For a pair of assigned ${ }^{12} \mathrm{C}$ and ${ }^{13} \mathrm{C}$ isotopic abundances, simulated ${ }^{12} \mathrm{C}^{12} \mathrm{C}$ and ${ }^{13} \mathrm{C}^{12} \mathrm{C}$ spectra were generated and summed. Continuum background emission at a level matching experimental results was also added. For the study of spectral interference, single or multiple spectral-interference peaks with tunable heights at variable wavelength positions were then added to the simulated $\mathrm{C}_{2}$ molecular spectrum. Similar to the case of molecular spectra, these interference line spectra also possess flicker noise. In our simulation, the flicker noise of the $\mathrm{C}_{2}$ molecular band and the spectral-interference peaks were uncorrelated and were independently generated. After the addition of interference peaks (if any), photon shot noise was added to individual data points in the simulated spectra. As expected, the photon shot noise was directly proportional to the square root of the simulated ICCD count rate; the proportionality constant was experimentally determined from the photon transfer curve of the ICCD. Furthermore, a single simulated spectrum can be used directly as in the case of singleshot LAMIS or can be accumulated to any desirable extent to mimic the situation of multiplepulse LAMIS.

Calibrations of simulated molecular spectra with predefined ${ }^{12} \mathrm{C}$ and ${ }^{13} \mathrm{C}$ isotopic abundances were performed with partial least squares (PLS) regression. The PLS algorithm used in this study was a PLS1 type and is similar to the one previously described $[2,10,11]$. A library of standard spectra from eleven (unless otherwise specified) standards with ${ }^{13} \mathrm{C}$ abundances evenly distributed between $0 \%$ and $2 \%$ was complied. For each standard, there were ten simulated spectra, and each spectrum comprised accumulated signals from 100 laser shots (unless otherwise specified). A separate set of sample spectra was also generated in the same fashion. For PLS calibration, it is usually necessary to pre-process the data before the regression [30]. The goal of pre-processing is to differentiate the data variations due to the changes in sample 
properties (isotopic abundance of carbon in the present case) versus those variations introduced into the experimental processes (e.g., signal fluctuations, noise). Normalization is a common data pre-processing process to reduce systematic variation and minimize the spectrum uncertainty before establishing the calibration model [10,30-32]. Accordingly, to emphasize the variations in the shape of the molecular spectra at different ${ }^{13} \mathrm{C}$ isotopic concentrations, all individual emission spectra were normalized to its norm $[10,33]$ to compensate for pulse-topulse variability. A PLS regression coefficient matrix, which correlates ${ }^{13} \mathrm{C}$ isotopic abundances to the relevant reference spectra, was then calculated from the standard spectra. To determine the ${ }^{13} \mathrm{C}$ isotopic abundance in the unknown sample, a mathematical inversion of the precomputed PLS coefficient matrix was applied to the sample spectra. All spectra simulation and PLS analyses were performed with in-house written LabVIEW programs (National Instruments).

\section{Results and Discussion}

\subsection{Regression coefficients and optimization of principal components in PLS calibration}

One crucial factor in PLS calibration is the optimization of the number of principal components (PC). The optimization was performed through a comparison of the root mean square error of prediction (RMSEP) against the number of PC involved in the model. The calibration set contained eleven standards with equally spaced ${ }^{13} \mathrm{C}$ abundances from $0 \%$ to $2 \%$. For each standard, ten replicated spectra were fed into the calibration model. A set of reference-sample spectra, consisting ten ${ }^{13} \mathrm{C}$ isotopic abundances evenly distributed from $0.1 \%$ to $1.9 \%$ was simulated. To obtain a representative statistics, 100 repeated measurements were made for each sample. For both the calibration standards and the "unknown" samples, the signal in each 
spectrum was an accumulation from 100 laser shots. The number of PC in the PLS calibration model was systematically varied from 1 to 10 . The same set of standard and sample spectra was used to ensure that any observed effects in the numbers of PC are not due to different noise behavior in the spectra. Figure $2 \mathrm{~b}$ presents the influence of the numbers of PC in the PLS calibration model on the statistical parameter RMSEP, which is defined as

$$
R M S E P=\sqrt{\frac{1}{N} \sum_{i=1}^{N}\left(Y_{i, S}-Y_{i, p}\right)^{2}}
$$

where $Y_{i, s}$ is the standard (i.e., known) concentration of the $i^{\text {th }}$ sample, $Y_{i, p}$ is the predicted concentration from the calibration model of the $i^{\text {th }}$ sample, and $N$ is the total number of prediction samples. A dramatic reduction in RMSEP is observed when the number of PC is raised from 1 to 2 . Increasing the number of PC to 3 brings a comparatively slight, yet notable, improvement. Further inclusions of more PC into the PLS calibration model provide no improvement to the analysis. Therefore, three principal components was used in all PLS calibration models in the present study. Because the information in the ${ }^{12} \mathrm{C}^{12} \mathrm{C}$ and ${ }^{13} \mathrm{C}^{12} \mathrm{C}$ are all correlated, the first principal component contains mostly the signals whereas other components are dominated by noise; therefore, the optimum number of PC might be expected to be one. This argument neglects the fact that noise in spectra produced from laser induced plasma also contains valuable information about the sample. Moon and Omenetto [34, 35] showed that, in many cases, noise patterns in LIBS spectra resemble the signals, and thus contain information. As most noise is contained in other PC, the optimum number of PC is higher than one. 
Figure 2c shows the regression coefficients of the PLS model with the optimum (i.e., three) number of PC. It illustrates the spectral decomposition power of PLS on highly overlapping peaks. Compared to the ${ }^{12} \mathrm{C}^{12} \mathrm{C}$ and ${ }^{13} \mathrm{C}^{12} \mathrm{C}$ spectra presented in Figure 2a, it is clear that substantially more positive coefficients are found at the three bandheads of ${ }^{13} \mathrm{C}^{12} \mathrm{C}$ (at 474.4 , 472.2 and $470.4 \mathrm{~nm}$ ), even though the ${ }^{13} \mathrm{C}^{12} \mathrm{C}$ peaks are roughly $50 \times$ (there is a statistical factor of 2 for ${ }^{13} \mathrm{C}^{12} \mathrm{C}$ ) weaker than that of ${ }^{12} \mathrm{C}^{12} \mathrm{C}$. Similarly, negative coefficients were observed at the ${ }^{12} \mathrm{C}^{12} \mathrm{C}$ bandheads.

\subsection{Ultimate analytical figures of merit for ${ }^{13} \mathrm{C}$ isotopic analysis by LAMIS with PLS calibration}

The accuracy and precision of LAMIS with PLS calibration were gauged by isotopic determination of a series of simulated reference samples. Figure 3 shows the determined ${ }^{13} \mathrm{C}$ isotopic abundances from a set of ten simulated reference-sample spectra. Similar to the simulation discussed in the last section, the ${ }^{13} \mathrm{C}$ isotopic abundances of the ten samples are evenly distributed between $0.1 \%$ and $1.9 \%$, whereas those for the eleven standards are equally spaced from $0 \%$ to $2 \%$. All signals were accumulated from 100 laser shots; 10 and 100 replicated spectra for each standard and samples, respectively, were simulated. It was found that PLS calibration is highly immune to both source-flicker and photon-shot noise. The simulated accuracies, in the absence of any spectral interference, are within $0.002 \%$ in absolute percentage abundance of ${ }^{13} \mathrm{C}$. The standard deviations of the determined isotopic abundances are practically independent of the isotopic abundance and are about $0.0011 \%$ to $0.0012 \%$ in absolute percentage abundance of ${ }^{13} \mathrm{C}$ for the studied ${ }^{13} \mathrm{C}$ range. Figure 3 also shows the precision, expressed as relative standard deviations in per-mille unit, of the determined ${ }^{13} \mathrm{C}$ abundance. As RSD can be 
viewed as expressing standard deviations in terms of the determined values of the measurements, the decreasing trend of the RSD presented in Figure 3 is merely a natural consequence of increasing ${ }^{13} \mathrm{C}$ isotopic abundance.

The effect of signal strength on the precision of LAMIS analysis was simulated. Figure 4 shows the precision, in terms of RSD of the determined ${ }^{13} \mathrm{C}$ isotopic abundance, as a function of lasershot accumulation for each measured spectrum. To simulate the effect of the number of laser pulses, a pool of individually generated spectra, each with noise individually added, was summed before feeding into the PLS model (i.e., analogue to the common practice in spectrum/laser-shot accumulation in LIBS). The PLS model is the same as described in the foregoing discussion. In order to obtain representative values for measurement precisions, 3000 replicated measurements were simulated (i.e., the simulation was repeated 3000 cycles and RSD was calculated from these 3000 readings). Due to random statistical nature of standard deviation, the sample size needs to be large or otherwise the calculated standard deviation is not representative. Our previous work [23] showed that 3000 cycles of measurement is a good compromise between being able to confidently estimate the measurement precision and computation time.

The ${ }^{13} \mathrm{C}$ abundance in this test sample was $1.1 \%$, and is close enough to simulate the results for samples with ${ }^{13} \mathrm{C}$ at natural abundance. The precision from PLS regression was found to obey the square-root law (slope of the log-log plot is -0.48 , cf. Figure 4) with the number of accumulated laser shots. As many factors could potentially affect the measured emission signals (e.g., emission light-collection efficiency, spectrometric efficiency of optical components, and chemical nature of the carbon samples $[17,36])$, the effect of precision against measured emission signals was also simulated (data not shown). As expected, precision varies inversely 
proportional to the square-root of the measured emission signals, indicating the importance of photon-shot noise in defining LAMIS precision.

With 100 laser shots (cf. Figure 4), the theoretical achievable precision (expressed as RSD) for ${ }^{13} \mathrm{C}$ abundance is approximately $1 \%$. Even with a single-shot measurement, the achievable precision is around $10 \%$ at ${ }^{13} \mathrm{C}$ natural abundance $(\sim 1.08 \%)$. Such a precision is sufficient to distinguish the ${ }^{13} \mathrm{C}$ isotopic variations in many naturally occurring carbon-bearing materials. For example, the variations of ${ }^{13} \mathrm{C}$ abundances in natural graphite are approximately 40\%o [37]. The simulation shows that the theoretical achievable precision of LAMIS should be able to differentiate graphite samples of different natural variations in ${ }^{13} \mathrm{C}$ abundances, with single laserpulse measurement.

It is instructive to compare the precision of isotopic analysis obtained from PLS calibration with wholly acquired spectrum versus that from simple single-pixel ratio of the bandhead heights. Due to its correlated nature, a substantial fraction of the source-flicker noise is cancelled out in the calculation of the ${ }^{13} \mathrm{C}^{12} \mathrm{C} /{ }^{12} \mathrm{C}^{12} \mathrm{C}$ ratio (i.e., isotopic ratio or abundance) because the ${ }^{12} \mathrm{C}^{12} \mathrm{C}$ and ${ }^{13} \mathrm{C}^{12} \mathrm{C}$ molecular bands are affected to the same extent (i.e., correlated) in a measurement under flicker noise. Nevertheless, source-flicker noise affects the precision of isotopic analysis indirectly through photon-shot noise, which is uncorrelated. As precision of isotopic abundance is dominant by photon-shot noise, a simple way to estimate the precision if the isotopic abundance is to be determined by the ratio of the bandhead heights is to compute the signal-toshot noise levels of the two bandheads. For a ${ }^{12} \mathrm{C}^{12} \mathrm{C}$ bandhead with $8.3 \times 10^{6}$ ICCD counts (cf. Figure 1), the signal-to-shot noise ratio is 439 (i.e., $\mathrm{RSD}=0.23 \%$ ). At an isotopic abundance of $1.1 \%$ for ${ }^{13} \mathrm{C}$, the corresponding height for the ${ }^{13} \mathrm{C}^{12} \mathrm{C}$ bandhead is $1.83 \times 10^{5}$ (with a statistical 
factor of 2 for ${ }^{13} \mathrm{C}^{12} \mathrm{C}$ ) ICCD counts; hence, its signal-to-shot noise ratio is 64.6 (i.e., RSD = $1.55 \%$ ). Because photon-shot noise is uncorrelated, it variance (i.e., square of uncertainty) adds up quadratically. Thus, the RSD of the ${ }^{13} \mathrm{C}^{12} \mathrm{C} /{ }^{12} \mathrm{C}^{12} \mathrm{C}$ ratio is $1.57 \%$, for a pair of single pixel measurement with a single laser shot. The precision obtained through PLS calibration with the wholly acquired spectrum is $\sim 1.0 \%$ (cf. Figure 4), which shows notable improvement over the ratio of the bandhead heights. The improvement is likely attributed to the fact that the wholly acquired spectrum was used in the PLS calibration. Further, the benefit of PLS calibration over univariate single-pixel ratio regression is that the latter works only under the condition that the two selected peaks for the two isotopes are free from spectral interference. In marked contrast, PLS calibration is able to identify the spectral features that do not correlate with the analyte (i.e., interference), and use other spectral regions that are comparatively free from interference for the analysis. Thus, PLS calibration functions well even when spectral interference is several times larger than the analyte peak, as will be discussed in Sections 3.4 and 3.5.

\subsection{Effect of number of standards employed in the calibration model}

The data presented in Figures 3 and 4 were simulated with eleven isotopic standards, with ${ }^{13} \mathrm{C}$ abundances varied linearly and distributed evenly between 0 to $2 \%$. However, isotope reference materials are quite limited and often an extensive series might not be readily available [38, 39]. Accordingly, the effect of the number of isotopic standards involved in the calibration model on analytical accuracy and precision was investigated. Figure 5 depicts the analytical performance of ${ }^{13} \mathrm{C}$ isotopic analysis when the calibration model contained two, three, five and eleven standards. The ${ }^{13} \mathrm{C}$ abundances were $0 \%$ and $2 \%$ for two-standard, $0 \%, 1 \%$ and $2 \%$ for threestandard, and $0 \%, 0.5 \%, 1 \%, 1.5 \%$ and $2 \%$ for five-standard calibrations. Regardless of the 
number of calibration standards, ten samples with ${ }^{13} \mathrm{C}$ abundances distributed evenly between $0.1 \%$ and $1.9 \%$ were simulated, each corresponded to a signal and noise levels equivalent to an accumulation of 100 laser shots. The differences between the determined and the known (i.e., true) ${ }^{13} \mathrm{C}$ abundances were plotted in Figure 5, with error bars representing the standard deviations of 100 repetitive simulations.

There are two noteworthy points in Figure 5. First, as expected, the analytical accuracy improves monotonically with increasing number of calibration standards. However, the analytical bias is very acceptable even when only two calibration standards were used to build the PLS calibration model. When the number of standards reduced from eleven to five, the effect on the calibration model is minimal and statistically insignificant compared to the error bars. When the number of standards further reduced to three, the analytical biases increased and were within $0.004 \%$ in absolute ${ }^{13} \mathrm{C}$ abundance for the studied range. Further reduction of the calibration standards to two, which is the bare minimum required for PLS calibration (PLS cannot operate with single-point calibration), causes the biggest jump in analytical bias; yet the largest bias is still around $0.008 \%$ in absolute ${ }^{13} \mathrm{C}$ abundance. The second observation is that the analytical precisions are both independent of the numbers of standards used and the ${ }^{13} \mathrm{C}$ isotopic abundances. In all simulated cases, the standard deviations, estimated from 100 repetitive measurements, were all about $0.001 \%$ in absolute ${ }^{13} \mathrm{C}$ abundance. Compared to the natural abundance of ${ }^{13} \mathrm{C}(\sim 1 \%)$, such a precision level is roughly $1 \%$ in terms of RSD. 


\subsection{Effect of single spectral interference peak on accuracy and precision}

Like any optical emission technique, spectral interferences could degrade the analytical performance of LAMIS. Purely based on probability, because the wavelength span of a molecular band is much wider than that of an atomic emission line, the likelihood of spectral interference in LAMIS is higher than in atomic emission spectrometry. The effect of spectral interferences on the analytical performance of LAMIS was also simulated in the present study. In this section, the investigated spectral interference was in the form of a single atomic emission line, with adjustable emission wavelength and strength. Spectral interferences from multiple atomic emission lines will be presented in the next section. Strength of spectral interference is expressed as the peak height ratios between the interference peak and the $(1-0)$ bandhead of ${ }^{12} \mathrm{C}_{2}$. Figure 6a shows a simulated spectrum of $C_{2}$ Swan band $(\Delta v=+1)$ with $1.1 \%{ }^{13} \mathrm{C}$ isotopic abundance and a hypothetical atomic emission line at $474.48 \mathrm{~nm}$ as spectral interference. In this particular example, the peak height of the interference peak is the same as that of the (1-0) bandhead of ${ }^{12} \mathrm{C}_{2}$, and its position is exactly at the (1-0) bandhead of ${ }^{12} \mathrm{C}^{13} \mathrm{C}$.

In our simulation, the interference peak is also present in the calibration standards; however, the flicker noise of the $\mathrm{C}_{2}$ molecular band and the spectral-interference peak were uncorrelated and were independently generated. The simulation is similar to the case when the sample is matrix matched but the ratios between the interference peak and the molecular band fluctuate on a shotto-shot basis. Figure $6 \mathrm{~b}$ shows the regression coefficients of the PLS calibration in the presence of spectral interference at the ${ }^{13} \mathrm{C}^{12} \mathrm{C}$ bandhead (cf. Figure 6a). The originally positive regression coefficient (cf. Figure 2c) at the ${ }^{13} \mathrm{C}^{12} \mathrm{C}$ bandhead at $474.4 \mathrm{~nm}$ declines when the interference is present (cf. Figure 6b), indicating that the PLS model correctly realize that the spectral 
interfering peak is not related to ${ }^{13} \mathrm{C}^{12} \mathrm{C}$. In this case, the determination of ${ }^{13} \mathrm{C}$ isotopic abundance is performed through the remaining spectral features that express positive regression coefficients (i.e., correlate with ${ }^{13} \mathrm{C}^{12} \mathrm{C}$ ).

Figure $6 \mathrm{c}$ shows the accuracy and precision of the determined ${ }^{13} \mathrm{C}$ abundances, under the influence of a spectral-interference peak at $474.48 \mathrm{~nm}$ (cf. Figure 6a), as a function of the ratios between the peak heights of the interference and ${ }^{12} \mathrm{C}_{2}(1-0)$ bandhead. Each measurement consisted of 3000 spectra, each with an accumulation of 100 laser shots. Figure $6 \mathrm{c}$ is plotted in double $y$-axes format; the additional $y$-axis on the right provides a direct view on both the relative accuracy and precision in per-mille unit. To elaborate, take the data point at 0.03 peak ratio as an example, the determined ${ }^{13} \mathrm{C}$ abundance (cf. the left axis) was about $(1.1024 \pm 0.0015) \%$. As the true ${ }^{13} \mathrm{C}$ abundance was exactly $1.1 \%$, the relative bias was about $2.2 \%$ and can be read directly from the right axis. The difference between the top of the error bar $(\sim 3.6 \%$, right axis) and the data point $(2.2 \%)$ is $1.4 \%$, which represents the RSD of the measurement.

We found that the pre-processing normalization procedure is crucial in minimizing the imperative effect of spectral interferences. In one approach, the norm $[10,33]$ of the whole spectrum (from 466 to $478 \mathrm{~nm}$ ) was used in the calculation of the normalization factor whereas only a selected portion of the spectrum (from 466 to $474 \mathrm{~nm}$, cf. Figure 6a) was employed in the second method. It should be stressed that, in the second method, although the interference peak was not included in determining the normalization factor, the whole spectrum (after normalization) from 466 to $478 \mathrm{~nm}$ was feed into the PLS regression for the ${ }^{13} \mathrm{C}$ abundance 
measurement. In any case, the same normalization approach was applied to all the spectra of both the standards and the samples.

Figure $6 \mathrm{c}$ shows the results from the two normalization methods, and it clearly demonstrates the importance of wavelength region selection for the calculation of the normalization factor. For the approach that utilizes the whole spectral window from 466 to $478 \mathrm{~nm}$, the precisions (error bars) start to increase when the peak height ratios reach 1. In contrast, the other normalization method, in which the spectral interfering peak is excluded, shows a significant degradation in precision only when the peak height ratios are greater than 10 . At height ratio of 30 , the precisions offered by the second method are worsened by about twice the case without any spectral interference. At height ratios $\geq 300$, the two methods offer comparable precisions. Proper choice of the wavelength range for the computation of the normalization factor increases the tolerance of spectral interference by at least one order of magnitude. At the maximum simulated peak ratio when the interference peak is $1000 \times$ stronger than the $(1-0)$ bandhead of ${ }^{12} \mathrm{C}_{2}$, the precision is predicted to degrade only by a factor of $6-$ from $\sim 1.3 \%$ in the absence of any spectral interference to $8 \%$. In every case, spectral interference affects only precision but not analytical accuracy, if the calibration standards are matrix matched. It should be stressed that only the calibration matrix needs to be matched but the noise patterns and fluctuations in the interference peak and the analyte emission do not need to be correlated.

The importance of excluding the spectral-interfering peak in the calculation of the normalization factor is further demonstrated in Figure 6d. Two relatively narrow spectral regions, one from 466 to $468 \mathrm{~nm}$ and the other from 473 to $474 \mathrm{~nm}$, were selected for the computation of normalization factor. These two regions, respectively, correspond to the far left side of the 
spectrum with relatively weak emission and the strong ${ }^{12} \mathrm{C}_{2}(1-0)$ bandhead. In this simulation, the center of the spectral interfering peak was scanned from 467 to $477 \mathrm{~nm}$. Peak ratio between the interfering peak and the reference ${ }^{12} \mathrm{C}_{2}(1-0)$ bandhead was 10 . Ten simulation cycles, each with a measurement of 3000 simulated spectra, were performed and the error bars represent the standard deviations of the simulated precisions. Clearly, whenever the interference peak enters the spectral region that is used for the calculation of the normalization factor, the analytical precisions drastically degrade. The interfering peak cannot be contained in the computation of normalization factor because its signal is uncorrelated to the emission signal of the molecular band. As the purpose of normalization is to differentiate the data variation due to the changes in isotopic composition against systematic variations due to experimental fluctuations and noise [10, 30-32], fluctuations (e.g., flicker and photon-shot noise) from the interference peak, if allowed to enter the computation of the normalization factor, weaken the purpose of normalization and degrade the precision.

\subsection{Effect of multiple interfering peaks on accuracy and precision}

In many cases, spectral interference appears in the form of multiple emission lines, either as different emission lines from a single interfering element or from multiple co-existing elements in the sample. To simulate the analytical performance of LAMIS under the influence of multiple-line spectral interference, atomic emission spectra from a common environmental sample matrix - soil - were simulated and added as spectral interference to the $\mathrm{C}_{2}$ molecular spectra. The simulated spectral interferences were based on experimentally measured LIBS spectra from a pressed pellet of soil sample (NIST SRM 2710a, described as Montana I soil). In the wavelength region from 466 to $478 \mathrm{~nm}$, four elements (Ti, Fe, Mn and Sr) were found to emit 
strongly and the Ti I $468.191 \mathrm{~nm}$ line was the strongest. A total of thirty-seven emission lines were identified with fifteen belong to Ti, ten each for Fe and Mn, and two for Sr (cf. Table 1 [40]). To simulate spectral interference from the soil matrix, the strongest LIBS emission lines for each element were first identified (lines marked with an asterisk in Table 1). Their experimentally measured ratios with the Ti I $468.191 \mathrm{~nm}$ line in the soil LIBS spectra were calculated; these ratios set the proportionality constants $(K)$ between emission lines from different elements in the simulation. The intensities $(I)$ of other atomic lines were then calculated, assuming a plasma temperature $(T)$ of $6000 \mathrm{~K}$, through the relationship:

$$
I=K \frac{(2 J+1) A}{Q(T)} e^{\frac{-E}{k T}}
$$

where $K$ is the proportionality constant relating emission between different elements, $J$ and $E$ are respectively the angular momentum and energy of the upper level of the atomic transition, $A$ is the transition probability, $Q(T)$ is the temperature-dependent partition function, and $k$ is the Boltzmann constant.

Similar to the case of a single spectral-interference line, the strength of the simulated spectral interference from soil was also adjustable and was expressed as the peak height ratios between the reference peak, Ti I $468.191 \mathrm{~nm}$, and the (1-0) bandhead of ${ }^{12} \mathrm{C}_{2}$. In addition, the flicker noise of the simulated soil spectra and the $\mathrm{C}_{2}$ molecular band were separately generated and thus uncorrelated. Figure 7a shows a simulated LIBS spectrum from soil matrix spectral interference on the $\mathrm{C}_{2}$ LAMIS spectrum. The ratio of spectral-interference peak (i.e., Ti I $468.191 \mathrm{~nm}$ ) to molecular band (i.e., (1-0) bandhead of ${ }^{12} \mathrm{C}_{2}$ ) was 1 in this example. As discussed in the last section, it is crucial to ensure that the normalized factor is computed from a spectral region that 
is free from interference. Accordingly, the wavelength region between 471.25 and $472 \mathrm{~nm}$ was selected for the computation of the normalization factor for simulated $\mathrm{C}_{2}$ spectra in soil matrix.

Figure $7 \mathrm{~b}$ shows the accuracy and precision of the determined ${ }^{13} \mathrm{C}$ abundances in the case of multiple-line spectral interference. The sample spectra were all based on $1.1 \%{ }^{13} \mathrm{C}$ abundance. PLS regression is impressive in differentiating the spectral patterns from the $\mathrm{C}_{2}$ emission versus those from spectral interferences. In all simulated cases, in which the maximum ratio of the strongest interference peak to the $\mathrm{C}_{2}$ bandhead was 1000 , the determined ${ }^{13} \mathrm{C}$ abundances agree with the true value within experimental uncertainty. The precisions, of course, worsen as the interference signals grow stronger. The precisions, expressed as RSD of the determined ${ }^{13} \mathrm{C}$ abundances, are relatively steady for peak ratios $\leq 1$ and are about 1 to $1.2 \%$. The precisions slightly increase to about $2 \%$ when the peak ratio becomes 3 , and further rise to $3 \%, 10 \%$ and $20 \%$ when the ratios are 10,100 and 1000, respectively. With spectral interference from multiple lines that many of their signals are hundreds times stronger than the analyte emission, the negligible predicted analytical bias and acceptable precision (only 20\% RSD) clearly reveal that PLS regression is a powerful and indispensable tool for extraction of isotopic information from LAMIS spectra.

\subsection{Effect of spectral resolution on precision}

The last simulation performed in this study was to understand the effect of spectral resolution on the analytical performance of LAMIS calibrated by PLS regression. Figure 8a shows the analytical accuracy and precision as a function of spectral resolution. The test sample contained $1.1 \%{ }^{13} \mathrm{C}$. Also, a single spectral-interference peak overlapping with the ${ }^{13} \mathrm{C}(1-0)$ bandhead at 
$474.48 \mathrm{~nm}$ was present (cf. Figure 6a). The ratios between this interference peak and the ${ }^{12} \mathrm{C}_{2}$ $(1-0)$ bandhead were 0 (i.e., no spectral interference), 1 and 10. The dependencies of both the analytical accuracy and precision as function of spectral resolution were found to be similar for the three cases. Figure $8 \mathrm{~b}$ presents the RSDs of the determined ${ }^{13} \mathrm{C}$ abundances in logarithm scale and the RSDs increase monotonically with spectral bandpass. The rise is gentle when the spectral bandpass is $500 \mathrm{pm}$ or less but becomes dramatic once the bandpass increases beyond $800 \mathrm{pm}$. When the spectral bandpass increases from $10 \mathrm{pm}$ to $400 \mathrm{pm}$ (a $40 \times$ change), the predicted precisions worsen by less than a factor of 2. In agreement with our previous report [2], the large isotope shift in molecular spectra together with the differentiating power of PLS regression relaxes the spectral resolution requirement for isotopic analysis and a spectrometer with resolution of a few $100 \mathrm{pm}$ can be employed. The relaxed spectral resolution requirement not only means that a reduction in the capital cost and footprint for the spectrometer, but a smaller spectrometer typically offers a higher light throughput. As shown in Section 3.2, precision improves when signal increases. Therefore, depending on the relative gain in light throughput, the slight negative effect through the use of a lower spectral resolution could potentially be balanced by the increase in throughput.

\section{Conclusion}

Through computer simulation, the fundamental analytical accuracy and precision attainable by LAMIS for minor isotopes at abundance levels between sub- and single-percentage were investigated. In the presence of measurement noise (e.g., source flicker, photon shot) but absence of spectral interference, the simulated accuracy and precision (standard deviation) expressed in units of absolute percentage of ${ }^{13} \mathrm{C}$ isotopic abundance can reach levels of $0.01 \%$ 
and $0.001 \%$, respectively, with the use of only two calibration standards. The influence of spectral interference depends on the ratio of the magnitudes between the interference and the analyte peaks. The effect from spectral interference, albeit minor, is noticeable when this ratio becomes one. An effective way to minimize the impact of spectral interference is to carefully select the wavelength region for the computation of the normalization factor; no spectralinterfering peak should be allowed to enter this computation or else analytical precision greatly degrades. For calibration with matrix-matched standards, spectral interference affects only precision but not analytical accuracy. Partial least square regression is an effectual filter for noise and spectral interferences. The present study offers a better understanding on the analytical performance of LAMIS, its limitation in the presence of spectral interferences, and ways to minimize their impacts.

\section{Acknowledgment}

This work was supported by the Defense Nuclear Nonproliferation Research and Development Office, and the Director, Office of Science, Office of Basic Energy Sciences, Division of Chemical Sciences, Geosciences, and Biosciences, Separations and Analysis Program of the U.S. Department of Energy under contract number DE-AC02-05CH11231 at the Lawrence Berkeley National Laboratory. 


\section{References}

1. X. Mao, A.A. Bol'shakov, I. Choi, C.P. McKay, D.L. Perry, O. Sorkhabi, R.E. Russo, Laser ablation molecular isotopic spectrometry: Strontium and its isotopes, Spectrochim. Acta Part B 66 (2011) 767-775.

2. X. Mao, A.A. Bol'shakov, D.L. Perry, O. Sorkhabi, R.E. Russo, Laser ablation molecular isotopic spectrometry: Parameter influence on boron isotope measurements, Spectrochim. Acta Part B 66 (2011) 604-609.

3. R.E. Russo, A.A. Bol'shakov, X. Mao, C.P. McKay, D.L. Perry, O. Sorkhabi, Laser ablation molecular isotopic spectrometry, Spectrochim. Acta Part B 66 (2011) 99-104.

4. H. Hou, G.C.Y. Chan, X. Mao, R. Zheng, V. Zorba, R.E. Russo, Femtosecond filament-laser ablation molecular isotopic spectrometry, Spectrochim. Acta Part B 113 (2015) 113-118.

5. A. Elliott, Determination of the abundance ratios of isotopes from band spectra, Nature 126 (1930) 845-846.

6. A. Elliott, Bestimmung des isotopenverhältnisses durch intensitätsmessungen im bormonoxyd-spektrum, Zeitschrift für Physik 67 (1931) 75-88.

7. H. Niki, T. Yasuda, I. Kitazima, Measurement technique of boron isotopic ratio by laserinduced breakdown spectroscopy, J. Nucl. Sci. Technol. 35 (1998) 34-39.

8. B. Yee, K.C. Hartig, P. Ko, J. McNutt, I. Jovanovic, Measurement of boron isotopic ratio with non-gated molecular spectroscopy of femtosecond laser-produced plasma, Spectrochim. Acta Part B 79-80 (2013) 72-76.

9. P. Ko, I. Jovanovic, Boron isotopic measurements from spectrally filtered non-gated molecular spectra induced by laser ablation, Spectrochim. Acta Part B 90 (2013) 68-71.

10. A. Sarkar, X. Mao, R.E. Russo, Advancing the analytical capabilities of laser ablation molecular isotopic spectrometry for boron isotopic analysis, Spectrochim. Acta Part B 92 (2014) 42-50.

11. A. Sarkar, X. Mao, G.C.Y. Chan, R.E. Russo, Laser ablation molecular isotopic spectrometry of water for ${ }_{1} \mathrm{D}^{2} /{ }_{1} \mathrm{H}^{1}$ ratio analysis, Spectrochim. Acta Part B 88 (2013) 46-53.

12. M. Berglund, M.E. Wieser, Isotopic compositions of the elements 2009 (IUPAC technical report), Pure Appl. Chem. 83 (2011) 397-410.

13. S. Brown, A. Ford, C.C. Akpovo, J. Martinez, L. Johnson, Matrix effects in laser ablation molecular isotopic spectrometry, Spectrochim. Acta Part B 101 (2014) 204-212. 
14. H. Hou, G.C.Y. Chan, X. Mao, V. Zorba, R. Zheng, R.E. Russo, Femtosecond laser ablation molecular isotopic spectrometry for zirconium isotope analysis, Anal. Chem. 87 (2015) 4788-4796.

15. E.V. Thomas, D.M. Haaland, Comparison of multivariate calibration methods for quantitative spectral-analysis, Anal. Chem. 62 (1990) 1091-1099.

16. D.M. Haaland, E.V. Thomas, Partial least-squares methods for spectral analyses .1. Relation to other quantitative calibration methods and the extraction of qualitative information, Anal. Chem. 60 (1988) 1193-1202.

17. S. Barbier, S. Perrier, P. Freyermuth, D. Perrin, B. Gallard, N. Gilon, Plastic identification based on molecular and elemental information from laser induced breakdown spectra: A comparison of plasma conditions in view of efficient sorting, Spectrochim. Acta Part B 88 (2013) 167-173.

18. S. Acquaviva, M.L. De Giorgi, High-resolution investigations of $\mathrm{C}_{2}$ and $\mathrm{CN}$ optical emissions in laser-induced plasmas during graphite ablation, J. Phys. B. 35 (2002) 795-806.

19. M.R. Dong, X.L. Mao, J.J. Gonzalez, J.D. Lu, R.E. Russo, Carbon isotope separation and molecular formation in laser-induced plasmas by laser ablation molecular isotopic spectrometry, Anal. Chem. 85 (2013) 2899-2906.

20. M. Dong, G.C.Y. Chan, X. Mao, J.J. Gonzalez, J. Lu, R.E. Russo, Elucidation of $\mathrm{C}_{2}$ and CN formation mechanisms in laser-induced plasmas through correlation analysis of carbon isotopic ratio, Spectrochim. Acta Part B 100 (2014) 62-69.

21. A.A. Bol'shakov, X. Mao, J. Jain, D.L. McIntyre, R.E. Russo, Laser ablation molecular isotopic spectrometry of carbon isotopes, Spectrochim. Acta Part B 113 (2015) 106-112.

22. J.M. Mermet, P. Mauchien, J.L. Lacour, Processing of shot-to-shot raw data to improve precision in laser-induced breakdown spectrometry microprobe, Spectrochim. Acta Part B 63 (2008) 999-1005.

23. G.C.Y. Chan, X. Mao, I. Choi, A. Sarkar, O.P. Lam, D.K. Shuh, R.E. Russo, Multiple emission line analysis for improved isotopic determination of uranium - a computer simulation study, Spectrochim. Acta Part B 89 (2013) 40-49.

24. R.P. Sperline, A.K. Knight, C.A. Gresham, D.W. Koppenaal, G.M. Hieftje, M.B. Denton, Read-noise characterization of focal plane array detectors via mean-variance analysis, Appl. Spectrosc. 59 (2005) 1315-1323. 
25. C.G. Parigger, A.C. Woods, D.M. Surmick, G. Gautam, M.J. Witte, J.O. Hornkohl, Computation of diatomic molecular spectra for selected transitions of aluminum monoxide, cyanide, diatomic carbon, and titanium monoxide, Spectrochim. Acta Part B 107 (2015) $132-138$.

26. R.S. Ram, J.S.A. Brooke, P.F. Bernath, C. Sneden, S. Lucatello, Improved line data for the Swan system ${ }^{12} \mathrm{C}^{13} \mathrm{C}$ isotopologue, Astrophys. J. Suppl. Ser. 211 (2014) 5.

27. J.S.A. Brooke, P.F. Bernath, T.W. Schmidt, G.B. Bacskay, Line strengths and updated molecular constants for the $\mathrm{C}_{2}$ Swan system, J. Quant. Spectrosc. Radiat. Transf. 124 (2013) $11-20$.

28. R.L. Kurucz, Diatomic molecular data for opacity calculations (Kurucz database CD-Rom \#15), Harvard-Smithsonian Center for Astrophysics, address: http://kurucz.harvard.edu/molecules.html, (accessed 27 Oct 2015).

29. C. Amiot, Fourier spectroscopy of the ${ }^{12} \mathrm{C}_{2},{ }^{13} \mathrm{C}_{2}$, and ${ }^{12} \mathrm{C}^{13} \mathrm{C}(0-0)$ Swan bands, Astrophys. J. Suppl. Ser. 52 (1983) 329-340.

30. M. Zeaiter, J.M. Roger, V. Bellon-Maurel, Robustness of models developed by multivariate calibration. Part II: The influence of pre-processing methods, Trends Anal. Chem. 24 (2005) 437-445.

31. J. Feng, Z. Wang, L. West, Z. Li, W. Ni, A PLS model based on dominant factor for coal analysis using laser-induced breakdown spectroscopy, Anal. Bioanal. Chem. 400 (2011) 3261-3271.

32. H. Feilhauer, G.P. Asner, R.E. Martin, S. Schmidtlein, Brightness-normalized partial least squares regression for hyperspectral data, J. Quant. Spectrosc. Radiat. Transf. 111 (2010) 1947-1957.

33. A. Sarkar, V. Karki, S.K. Aggarwal, G.S. Maurya, R. Kumar, A.K. Rai, X. Mao, R.E. Russo, Evaluation of the prediction precision capability of partial least squares regression approach for analysis of high alloy steel by laser induced breakdown spectroscopy, Spectrochim. Acta Part B 108 (2015) 8-14.

34. H.Y. Moon, Diagnostic and analytical studies of laser induced plasmas, University of Florida, Ph.D. Thesis (2010). 
35. D.W. Hahn, N. Omenetto, Laser-induced breakdown spectroscopy (LIBS), Part II: Review of instrumental and methodological approaches to material analysis and applications to different fields, Appl. Spectrosc. 66 (2012) 347-419.

36. J. Anzano, R.-J. Lasheras, B. Bonilla, J. Casas, Classification of polymers by determining of $\mathrm{C}_{1}: \mathrm{C}_{2}: \mathrm{CN}: \mathrm{H}: \mathrm{N}: \mathrm{O}$ ratios by laser-induced plasma spectroscopy (LIPS), Polymer Testing 27 (2008) 705-710.

37. T.B. Coplen, J.K. Bohlke, P. De Bievre, T. Ding, N.E. Holden, J.A. Hopple, H.R. Krouse, A. Lamberty, H.S. Peiser, K. Revesz, S.E. Rieder, K.J.R. Rosman, E. Roth, P.D.P. Taylor, R.D. Vocke, Y.K. Xiao, Isotope-abundance variations of selected elements (IUPAC technical report), Pure Appl. Chem. 74 (2002) 1987-2017.

38. W.A. Brand, T.B. Coplen, J. Vogl, M. Rosner, T. Prohaska, Assessment of international reference materials for isotope-ratio analysis (IUPAC technical report), Pure Appl. Chem. 86 (2014) 425-467.

39. J. Vogl, M. Rosner, W. Pritzkow, The need for new isotope reference materials, Anal. Bioanal. Chem. 405 (2013) 2763-2770.

40. P.L. Smith, C. Heise, J.R. Esmond, R.L. Kurucz, Kurucz atomic line database, HarvardSmithsonian Center for Astrophysics, address: http://www.pmp.uni-hannover.de/cgibin/ssi/test/kurucz/sekur.html, (accessed 11 Nov 2015). 
Table 1 Emission lines and their spectroscopic constants [40] for simulation of multiple-line spectral interferences.

\begin{tabular}{|c|c|c|c|c|c|c|}
\hline Spectral line & $A\left(\mathrm{~s}^{-1}\right)$ & $E_{\text {lower }}\left(\mathrm{cm}^{-1}\right)$ & $J_{\text {lower }}$ & $E_{\text {upper }}\left(\mathrm{cm}^{-1}\right)$ & $J_{\text {upper }}$ & $\begin{array}{l}\text { Relative } \\
\text { emission }\end{array}$ \\
\hline Fe I 467.316 & $4.57 \times 10^{6}$ & 29469.0 & 2 & 50861.8 & 3 & 0.0691 \\
\hline $\mathrm{Fe}$ I 467.885 & $7.40 \times 10^{6}$ & 29056.3 & 3 & 50423.1 & 4 & 0.1598 \\
\hline $\mathrm{Fe}$ I 469.141 & $1.19 \times 10^{6}$ & 24118.8 & 4 & 45428.4 & 4 & 0.0848 \\
\hline $\mathrm{Fe}$ I 470.727 & $2.78 \times 10^{6}$ & 26140.2 & 3 & 47378.0 & 4 & 0.1235 \\
\hline Fe I 470.909 & $5.06 \times 10^{6}$ & 29469.0 & 2 & 50698.6 & 2 & 0.0565 \\
\hline $\mathrm{Fe}$ I 471.028 & $9.99 \times 10^{5}$ & 24338.8 & 3 & 45563.0 & 3 & 0.0533 \\
\hline Fe I 472.740 & $9.78 \times 10^{6}$ & 29732.7 & 1 & 50880.1 & 1 & 0.0625 \\
\hline $\mathrm{Fe}$ I 473.359 & $6.45 \times 10^{4}$ & 11976.2 & 4 & 33095.9 & 4 & 0.0865 \\
\hline $\mathrm{Fe}$ I 473.677* & $4.92 \times 10^{6}$ & 25900.0 & 4 & 47005.5 & 5 & 0.2900 \\
\hline $\mathrm{Fe}$ I 474.580 & $6.86 \times 10^{6}$ & 29469.0 & 2 & 50534.4 & 3 & 0.1106 \\
\hline Ti I 467.512 & $1.85 \times 10^{6}$ & 8602.3 & 2 & 29986.2 & 3 & 0.0698 \\
\hline Ti I 468.191* & $2.35 \times 10^{6}$ & 386.9 & 4 & 21739.7 & 5 & 1.0000 \\
\hline Ti I 469.134 & $6.25 \times 10^{6}$ & 8602.3 & 2 & 29912.3 & 3 & 0.2399 \\
\hline Ti I 469.691 & $3.21 \times 10^{7}$ & 17369.6 & 1 & 38654.2 & 1 & 0.0652 \\
\hline Ti I 469.877 & $1.05 \times 10^{7}$ & 8492.4 & 1 & 29768.7 & 2 & 0.2970 \\
\hline Ti I 469.884 & $3.10 \times 10^{7}$ & 17423.9 & 2 & 38699.8 & 2 & 0.1040 \\
\hline Ti I 471.019 & $4.93 \times 10^{7}$ & 17540.2 & 3 & 38764.8 & 3 & 0.2269 \\
\hline Ti I 471.019 & $5.32 \times 10^{6}$ & 8436.6 & 0 & 29661.2 & 1 & 0.0924 \\
\hline Ti I 472.261 & $4.66 \times 10^{6}$ & 8492.4 & 1 & 29661.2 & 1 & 0.0808 \\
\hline Ti I 472.317 & $2.27 \times 10^{6}$ & 8602.3 & 2 & 29768.7 & 2 & 0.0640 \\
\hline Ti I 473.117 & $1.29 \times 10^{7}$ & 17540.2 & 3 & 38670.7 & 4 & 0.0776 \\
\hline Ti I 473.341 & $1.52 \times 10^{7}$ & 17423.9 & 2 & 38544.4 & 3 & 0.0732 \\
\hline Ti I 474.279 & $5.34 \times 10^{7}$ & 18037.2 & 4 & 39116.0 & 4 & 0.2887 \\
\hline Ti I 475.812 & $7.12 \times 10^{7}$ & 18141.2 & 5 & 39152.1 & 5 & 0.4651 \\
\hline Ti I 475.927 & $7.39 \times 10^{7}$ & 18192.6 & 6 & 39198.3 & 6 & 0.5643 \\
\hline Mn I 467.167 & $1.09 \times 10^{6}$ & 23296.7 & 3.5 & 44696.3 & 2.5 & 0.0065 \\
\hline Mn I 470.113 & $1.69 \times 10^{6}$ & 23549.2 & 2.5 & 44814.7 & 1.5 & 0.0065 \\
\hline
\end{tabular}




\begin{tabular}{lllllll} 
Mn I 470.971 & $1.72 \times 10^{7}$ & 23296.7 & 3.5 & 44523.4 & 3.5 & 0.1410 \\
Mn I 472.746 & $1.69 \times 10^{7}$ & 23549.2 & 2.5 & 44696.3 & 2.5 & 0.0992 \\
Mn I 473.909 & $2.40 \times 10^{7}$ & 23719.5 & 1.5 & 44814.7 & 1.5 & 0.0914 \\
Mn I 475.404 & $3.03 \times 10^{7}$ & 18402.5 & 2.5 & 39431.3 & 3.5 & 0.8313 \\
Mn I 476.151 & $5.35 \times 10^{7}$ & 23818.9 & 0.5 & 44814.7 & 1.5 & 0.2027 \\
Mn I 476.237* & $7.82 \times 10^{7}$ & 23296.7 & 3.5 & 44288.8 & 4.5 & 0.8400 \\
Mn I 476.585 & $4.07 \times 10^{7}$ & 23719.5 & 1.5 & 44696.3 & 2.5 & 0.2377 \\
Mn I 476.642 & $4.62 \times 10^{7}$ & 23549.2 & 2.5 & 44523.4 & 3.5 & 0.3748 \\
Sr I 472.227* & $4.43 \times 10^{7}$ & 14504.4 & 1 & 35674.7 & 2 & 0.6800 \\
Sr I 474.192 & $4.73 \times 10^{7}$ & 14317.5 & 0 & 35400.1 & 1 & 0.0779 \\
\hline
\end{tabular}

*strongest line for that particular element 


\section{Figure Captions}

Figure 1 Histogram showing experimental shot-to-shot fluctuations of peak heights of the ${ }^{12} \mathrm{C}^{12} \mathrm{C}\left(d^{3} \Pi_{\mathrm{g}}-a^{3} \Pi_{\mathrm{u}}, 1-0\right)$ bandhead at $473.73 \mathrm{~nm}$. The emission can be well represented by a Gaussian distribution (the solid line) with a relative standard deviation of $24 \%$.

Figure 2 (a) Simulated spectra of ${ }^{12} \mathrm{C}^{12} \mathrm{C}$ and ${ }^{13} \mathrm{C}^{12} \mathrm{C}$, and their sum with plasma continuum and noise. (b) Root mean square error of prediction (RMSEP) as a function of number of principal components in the PLS calibration model. Ten simulation cycles were performed, and their standard deviations are represented by the error bars. (c) Regression coefficient of the PLS calibration model (optimized, with 3 principal components) as a function of wavelength.

Figure 3 Isotopic abundances of ${ }^{13} \mathrm{C}$ determined by PLS calibration (left axis) and analytical precision as RSD in per-mille unit (right axis) against known ${ }^{13} \mathrm{C}$ abundances in the simulation. The calibration set contained eleven standards with equally spaced ${ }^{13} \mathrm{C}$ abundances from $0 \%$ to $2 \%$. All simulated spectra (both the samples and the calibration standards) were accumulated from 100 laser shots.

Figure 4 Relative standard deviations (RSD) of the determined ${ }^{13} \mathrm{C}$ isotopic abundances as a function of laser-shot accumulation in the measured LAMIS spectrum. The simulated sample contained $1.1 \%{ }^{13} \mathrm{C}$. A total of 3000 simulations were performed to determine the RSD.

Figure 5 Effect of the number of calibration standards in the PLS regression model on the analytical accuracy and precision of LAMIS. Isotopic abundances of ${ }^{13} \mathrm{C}$ in all calibration standards were equally spaced between $0 \%$ and $2 \%$. The error bar 
represents the standard deviation from 100 repetitive simulations, each with a 100 laser-shot accumulation.

Figure 6 (a) Simulated $\mathrm{C}_{2}$ LAMIS spectrum with a spectral-interference peak at $474.48 \mathrm{~nm}$, overlapping with the bandhead of ${ }^{12} \mathrm{C}^{13} \mathrm{C}$. In general, both the position and the strength of the interference peak are adjustable. In this example, the ratio of the interference peak and the ${ }^{12} \mathrm{C}_{2}(1-0)$ bandhead at $473.73 \mathrm{~nm}$ is 1 . (b) Regression coefficient of the PLS calibration model in the presence of a single-line spectral interference. (c) The determined ${ }^{13} \mathrm{C}$ abundance (left axis), and the relative accuracy (bias) and precision (as RSD) in per-mille unit (right axis) as a function of peak height ratios of the interference peak and the reference ${ }^{12} \mathrm{C}_{2}(1-0)$ bandhead. The simulated sample contained $1.1 \%{ }^{13} \mathrm{C}$, and a total of 3000 simulations were performed. (d) Analytical precision against the center wavelength of the interference peak and the spectral window for the calculation of the normalization factor. The error bar represents standard deviation from ten cycles of simulations.

Figure 7 (a) Simulated $\mathrm{C}_{2}$ LAMIS spectrum with multiple-line spectral interference from soil matrix. In this example, the ratio of the strongest interference peak (Ti I $468.191 \mathrm{~nm}$ ) and the ${ }^{12} \mathrm{C}_{2}(1-0)$ bandhead is 1 . (b) The determined ${ }^{13} \mathrm{C}$ abundance (left axis), and the relative accuracy (bias) and precision (as RSD) in per-mille unit (right axis) as a function of the strength of multiple-line spectral interference. The simulated sample contained $1.1 \%{ }^{13} \mathrm{C}$, and a total of 3000 simulations were performed.

Figure 8 (a) The determined ${ }^{13} \mathrm{C}$ abundance (left axis), and the relative accuracy (bias) and precision (as RSD) in per-mille unit (right axis) as a function of spectral resolution 
under the influence of single spectral-line interference at $474.48 \mathrm{~nm}$ at three different strengths. The simulated sample contained $1.1 \%{ }^{13} \mathrm{C}$, and a total of 3000 simulations were performed. All spectra were normalized with the norm from 466 to $474 \mathrm{~nm}$, prior to PLS regression. (b) Analytical precision as a function of spectral resolution. The error bar represents standard deviation from ten cycles of simulations. 
Figure 1

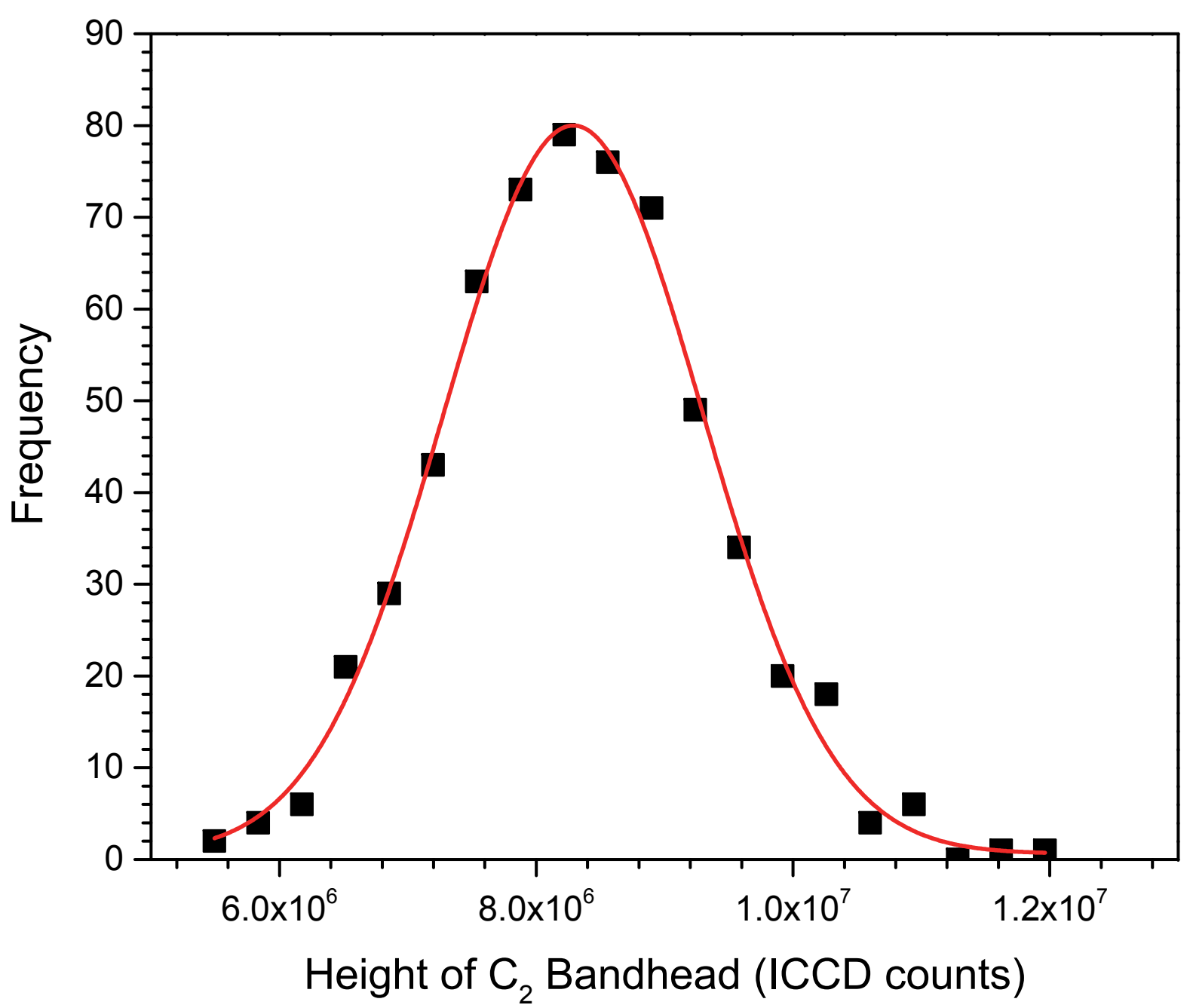


Figure $2 \mathrm{a}$

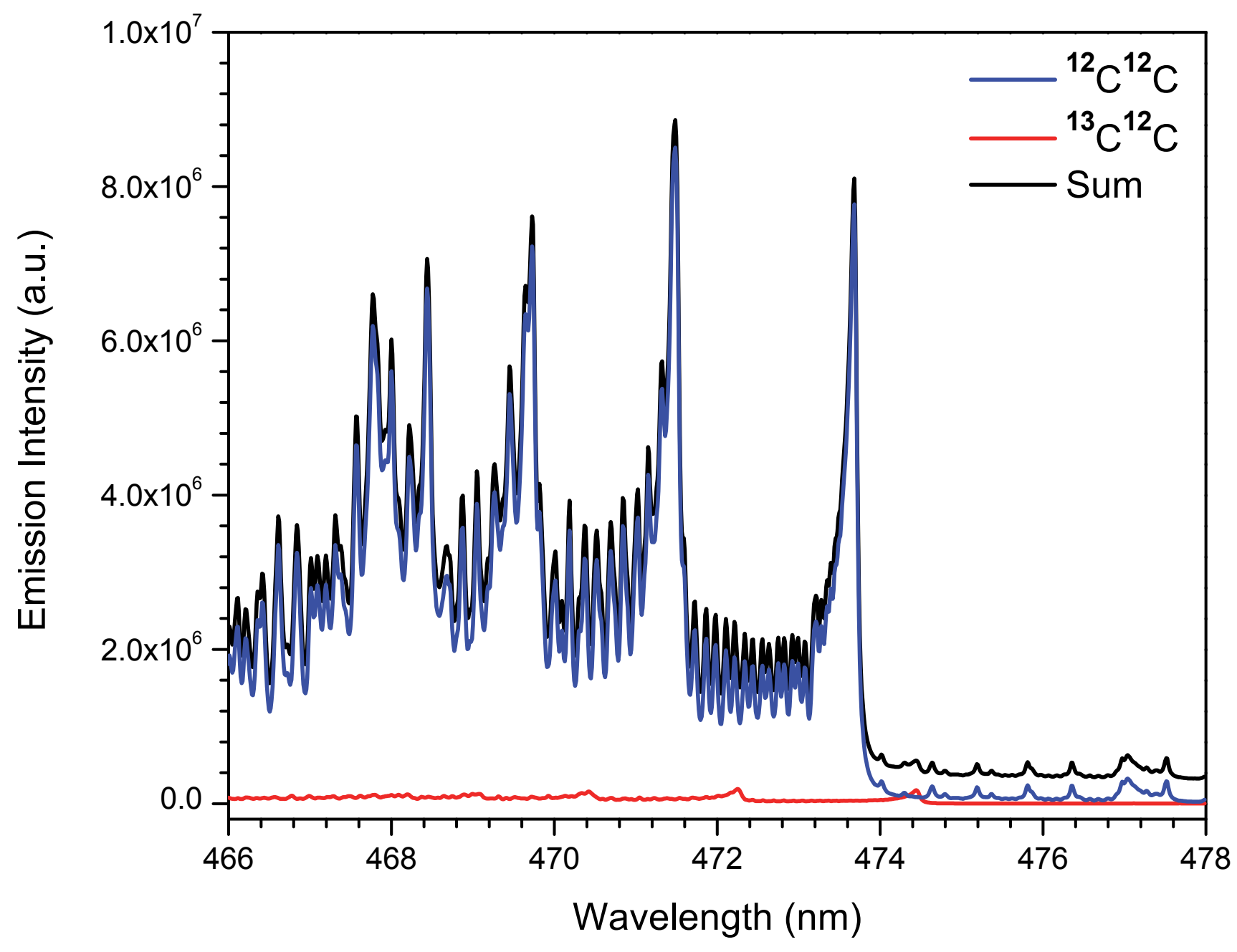


Figure $2 b$

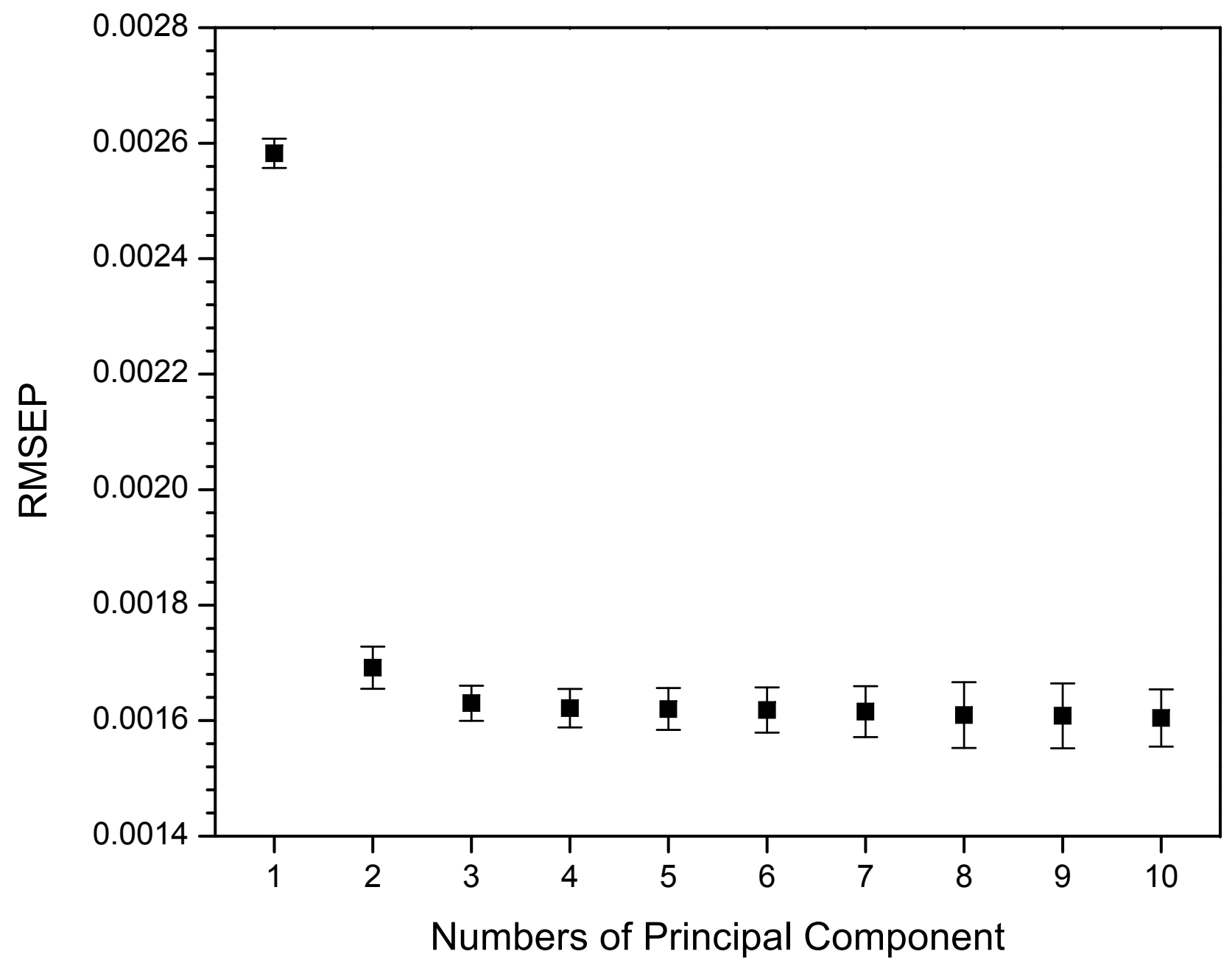


Figure 2c

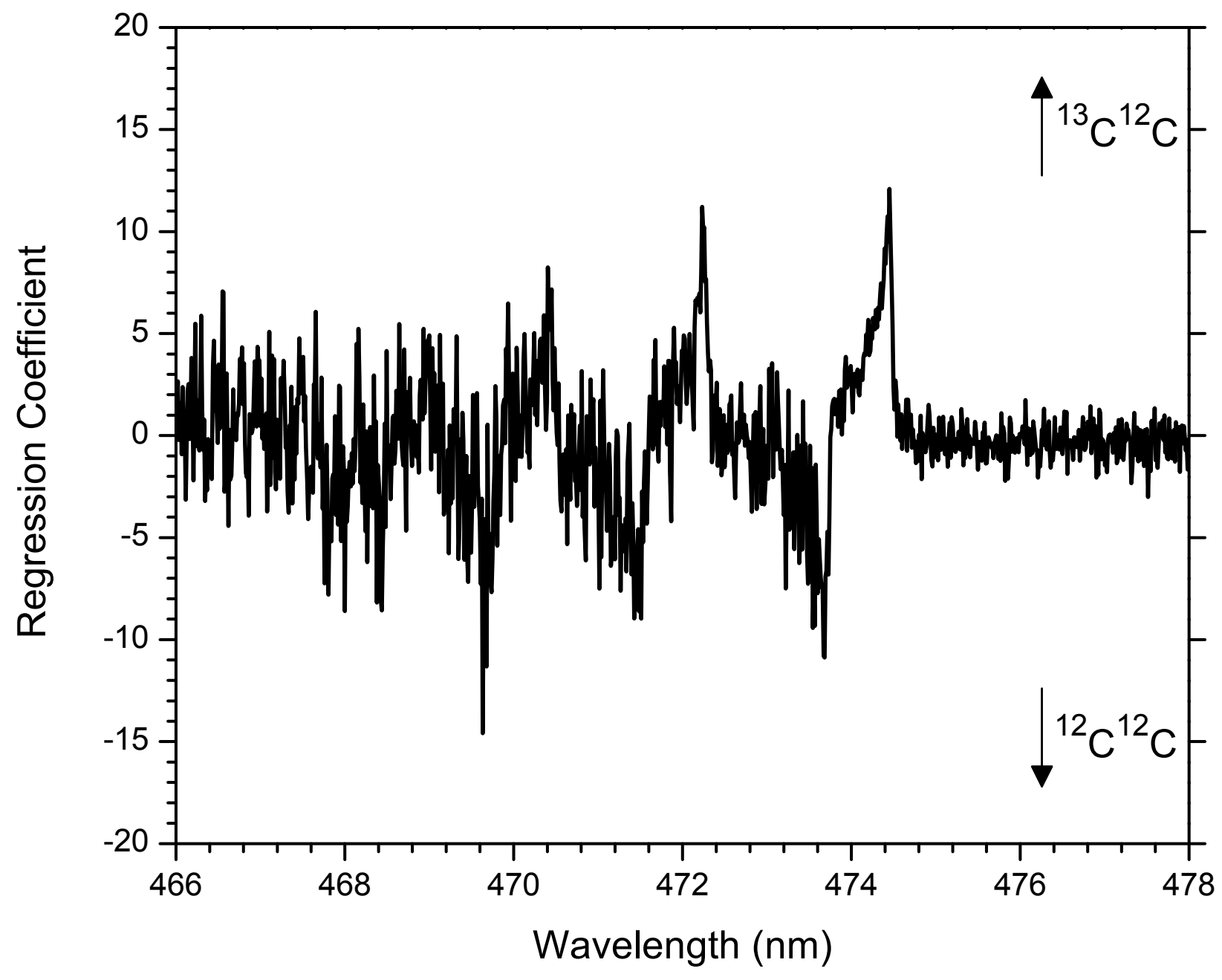


Figure 3

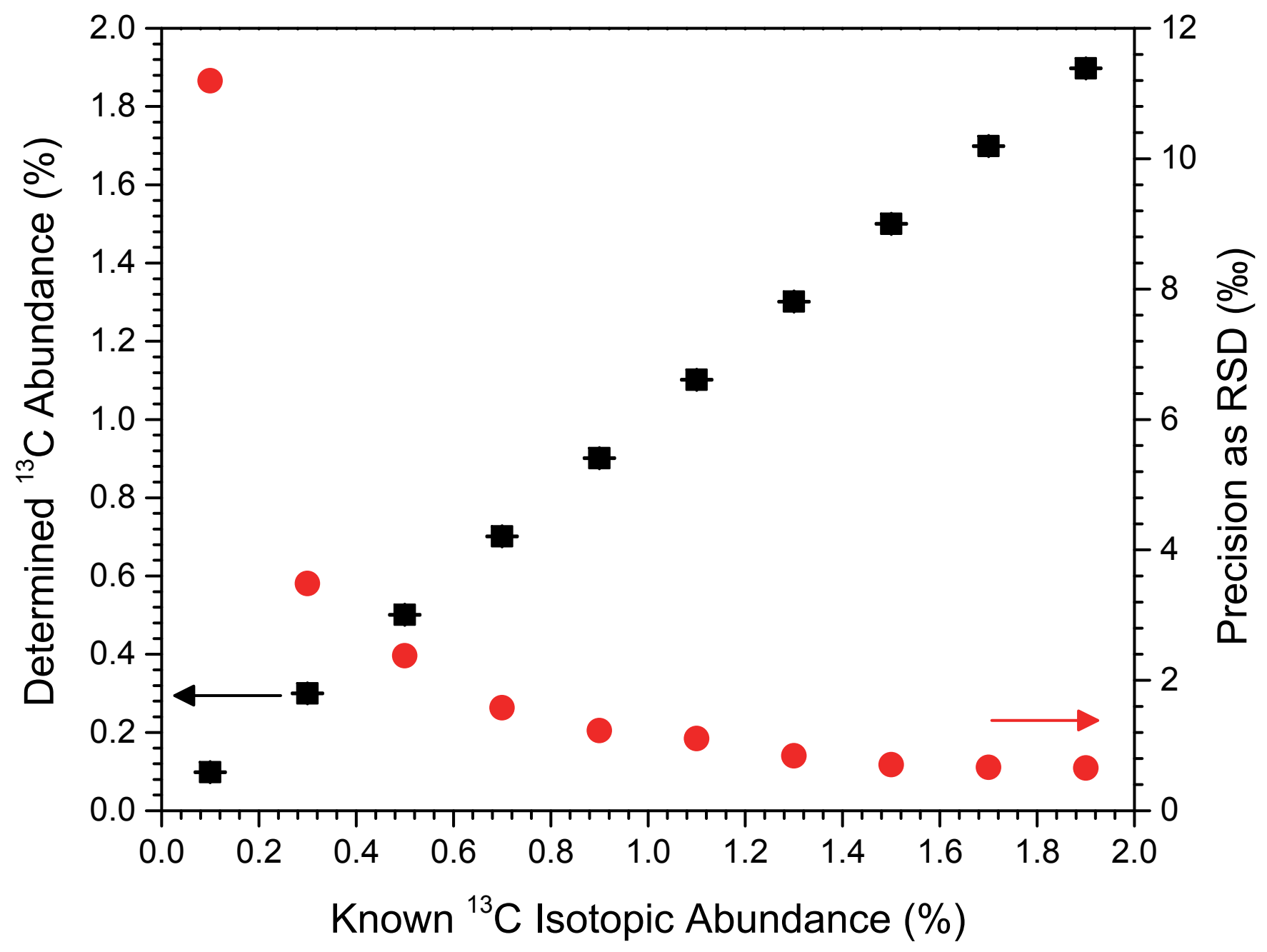


Figure 4

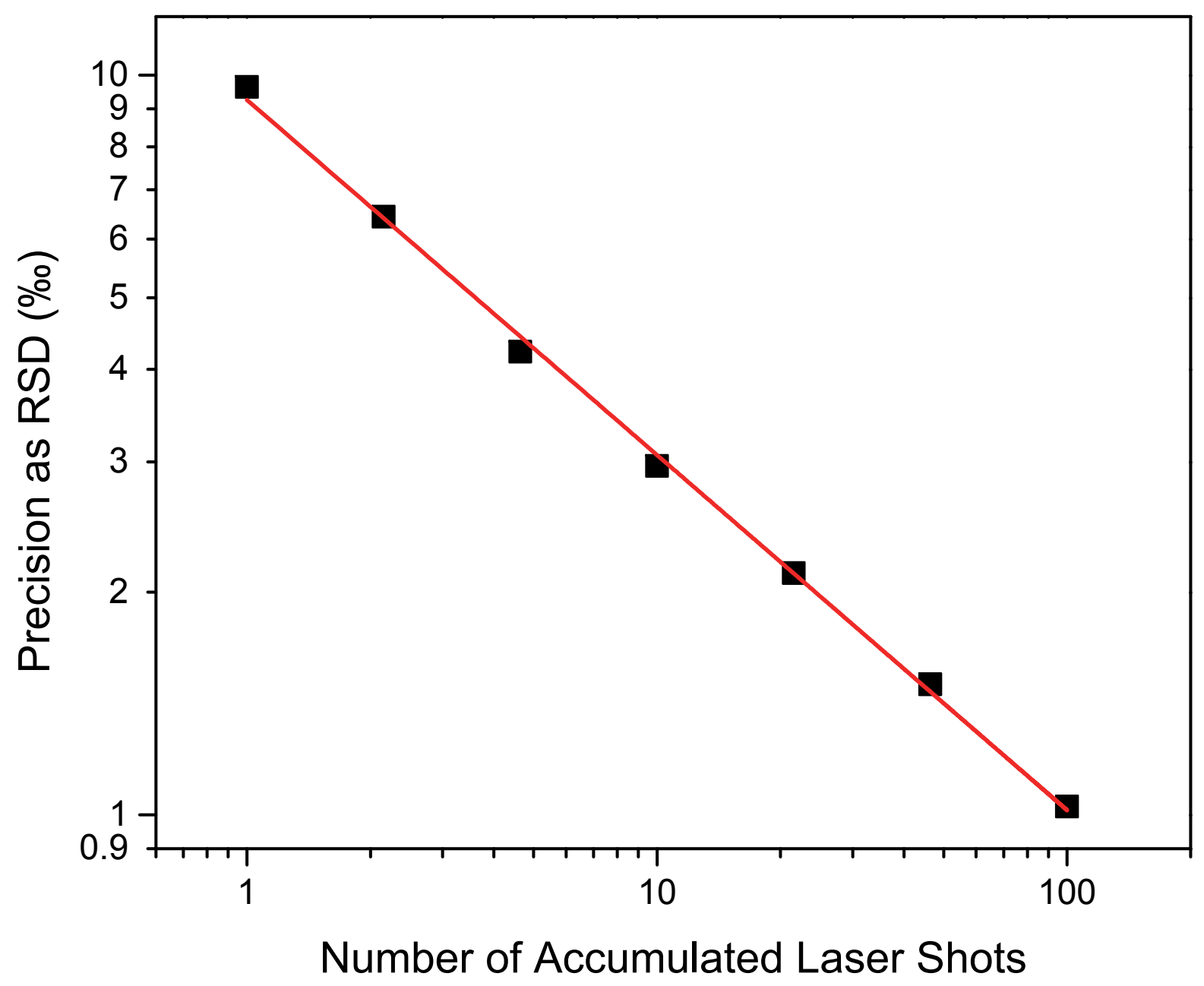


Figure 5

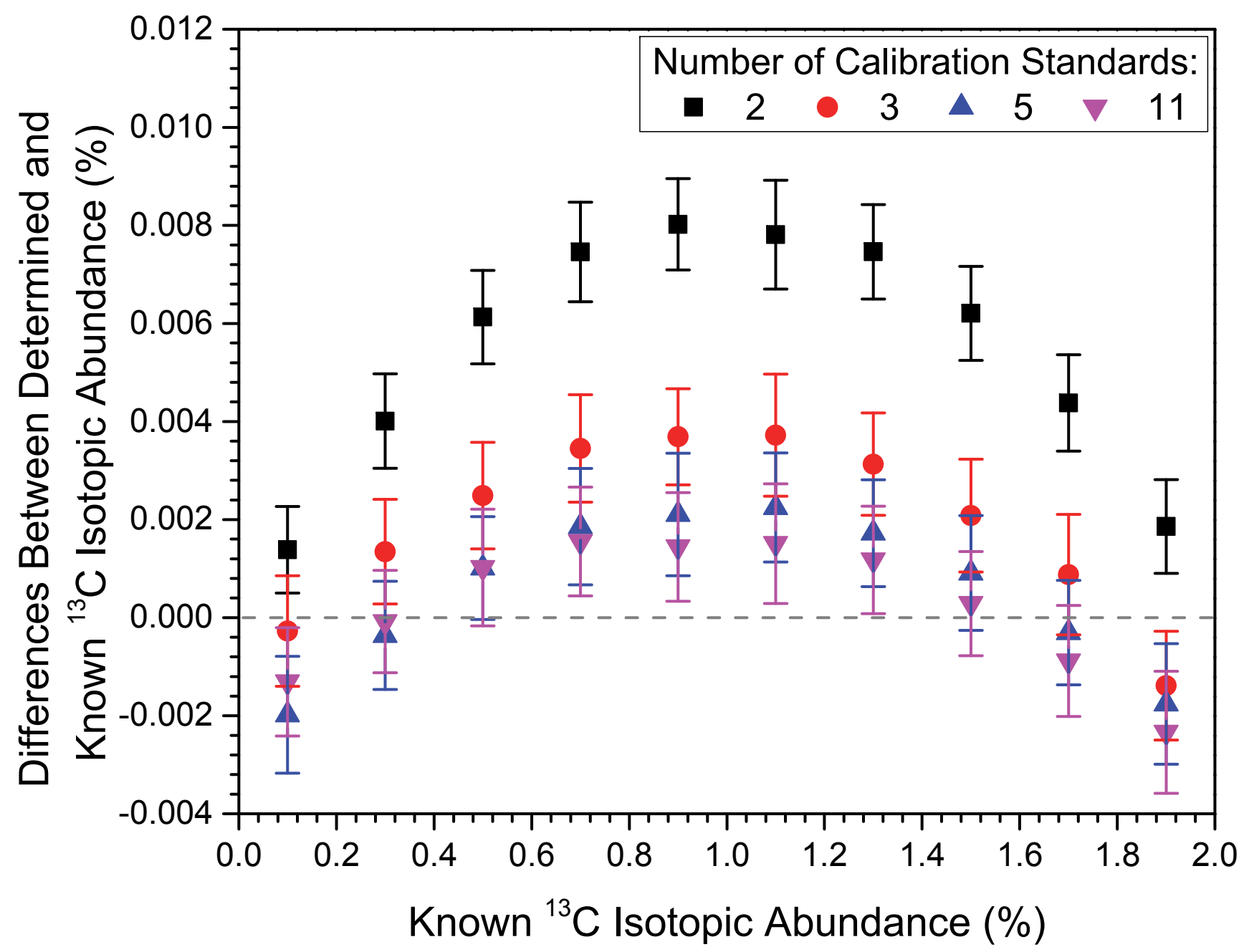


Figure 6a

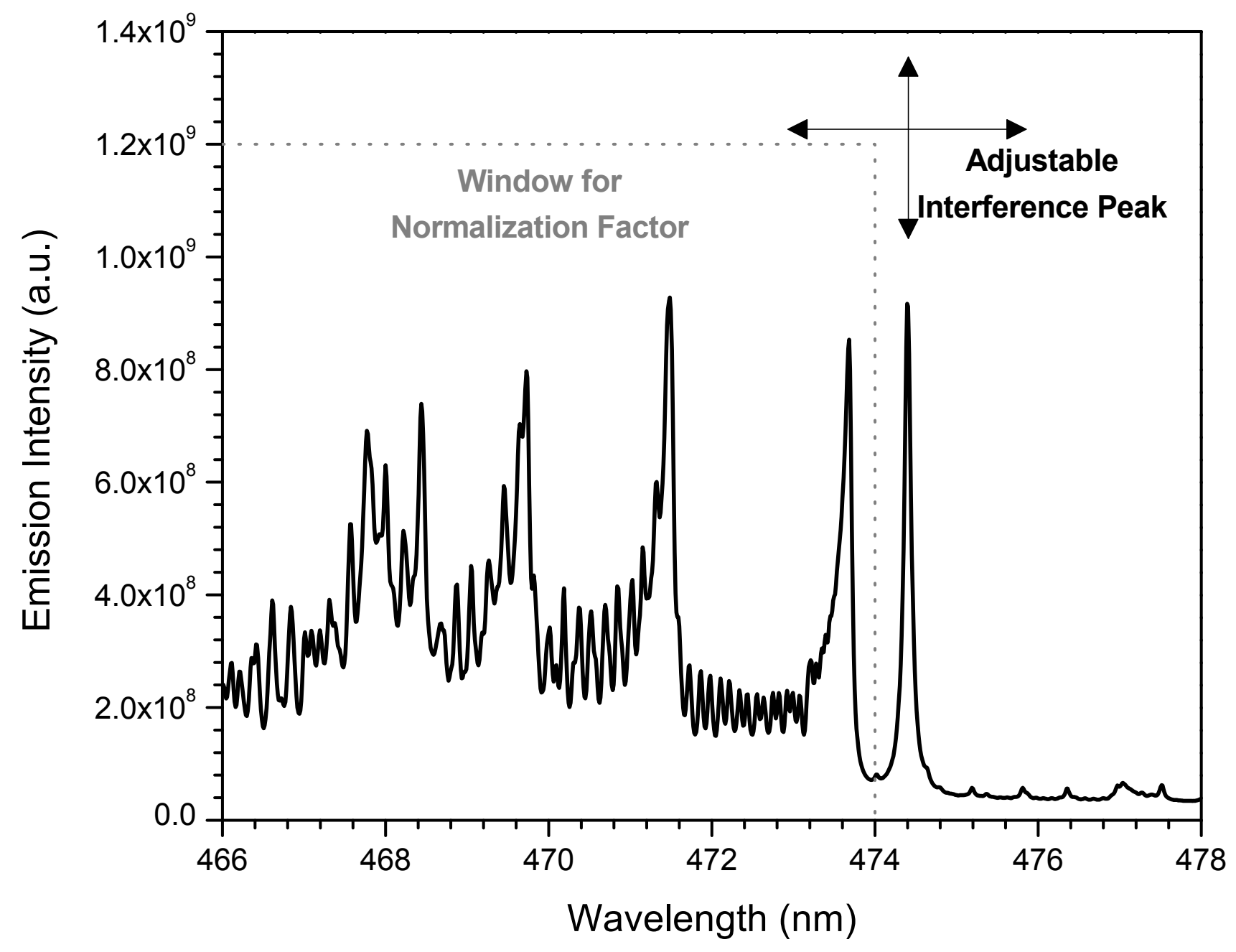


Figure 6b

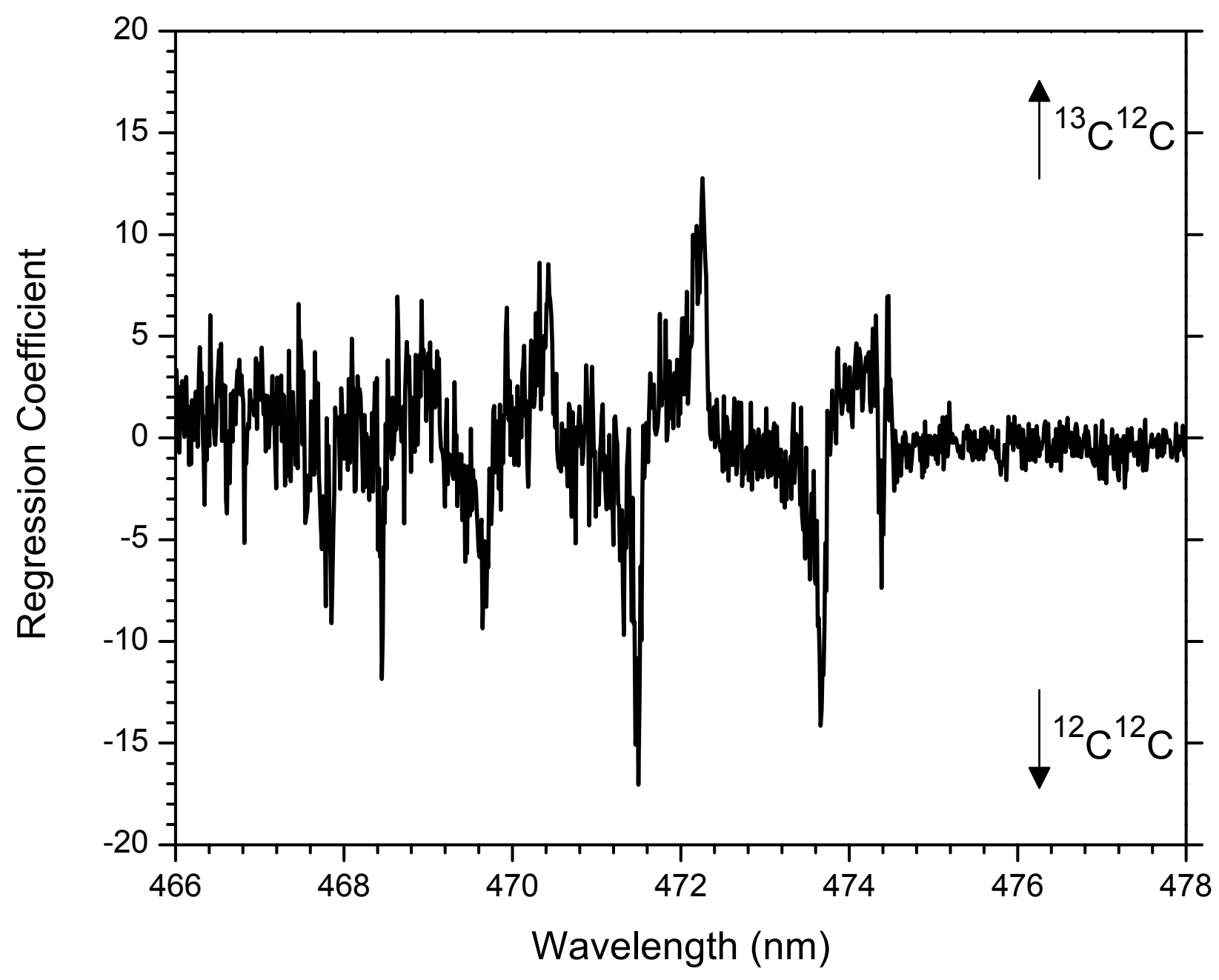


Figure 6c

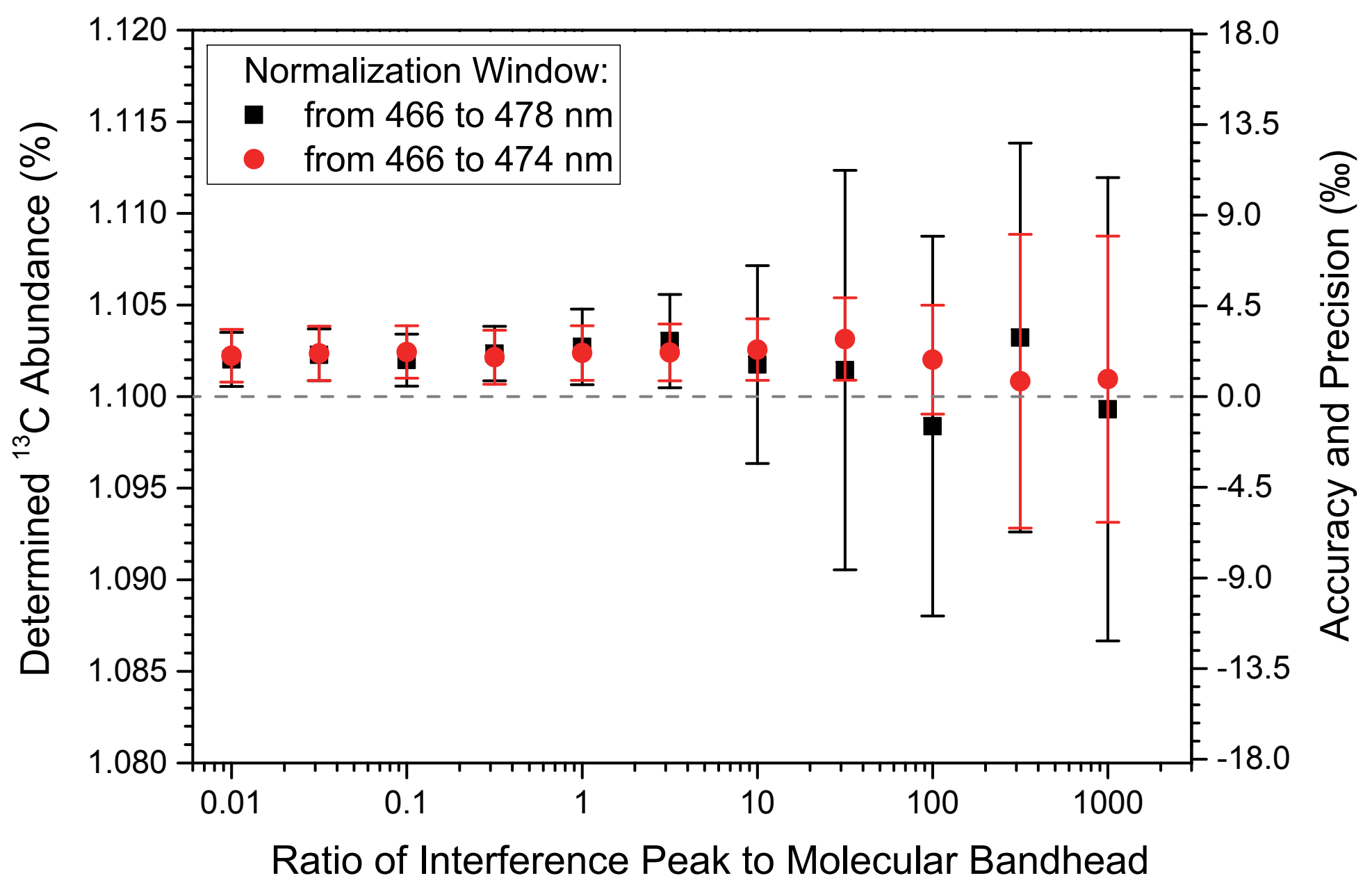




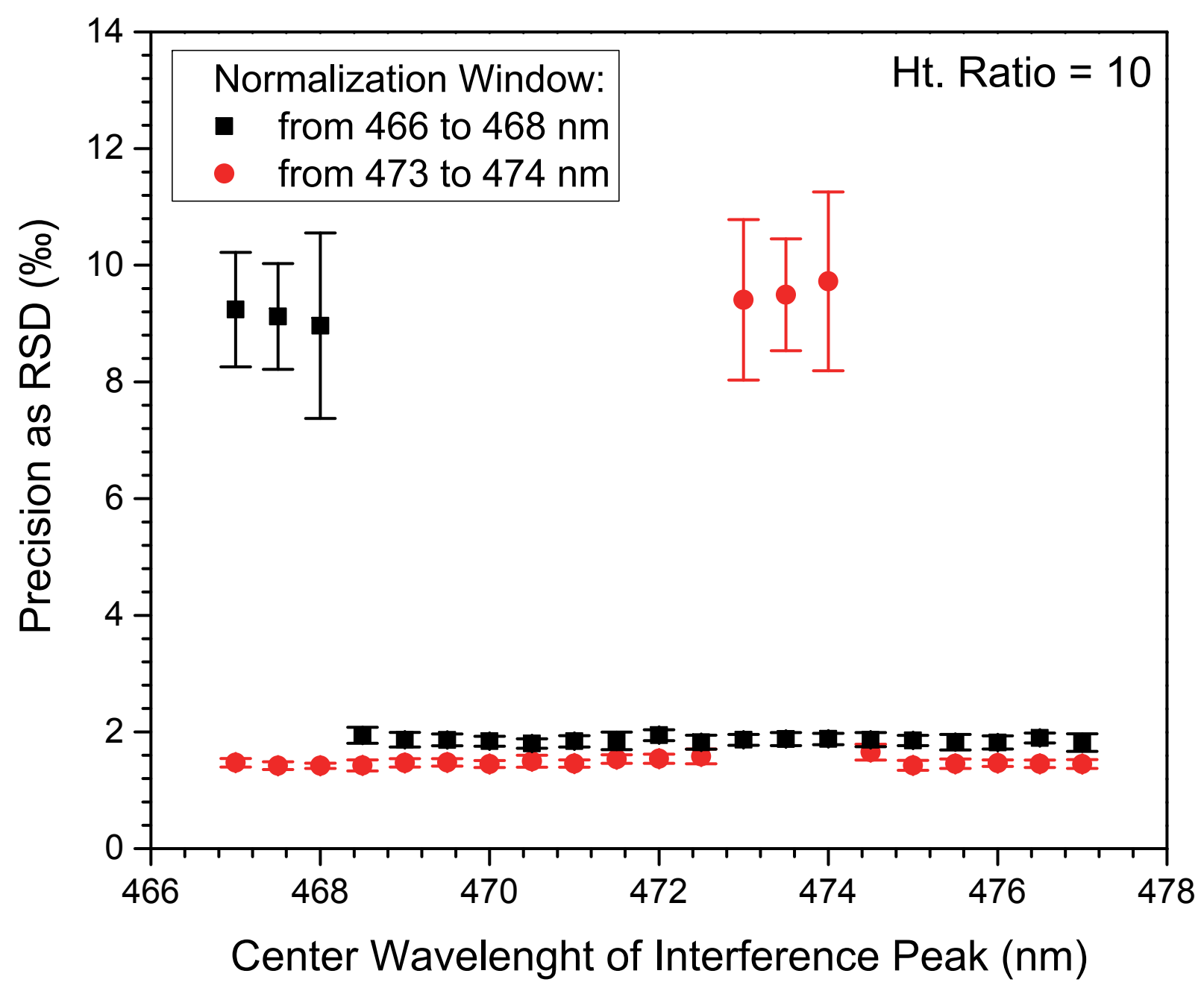


Figure $7 a$

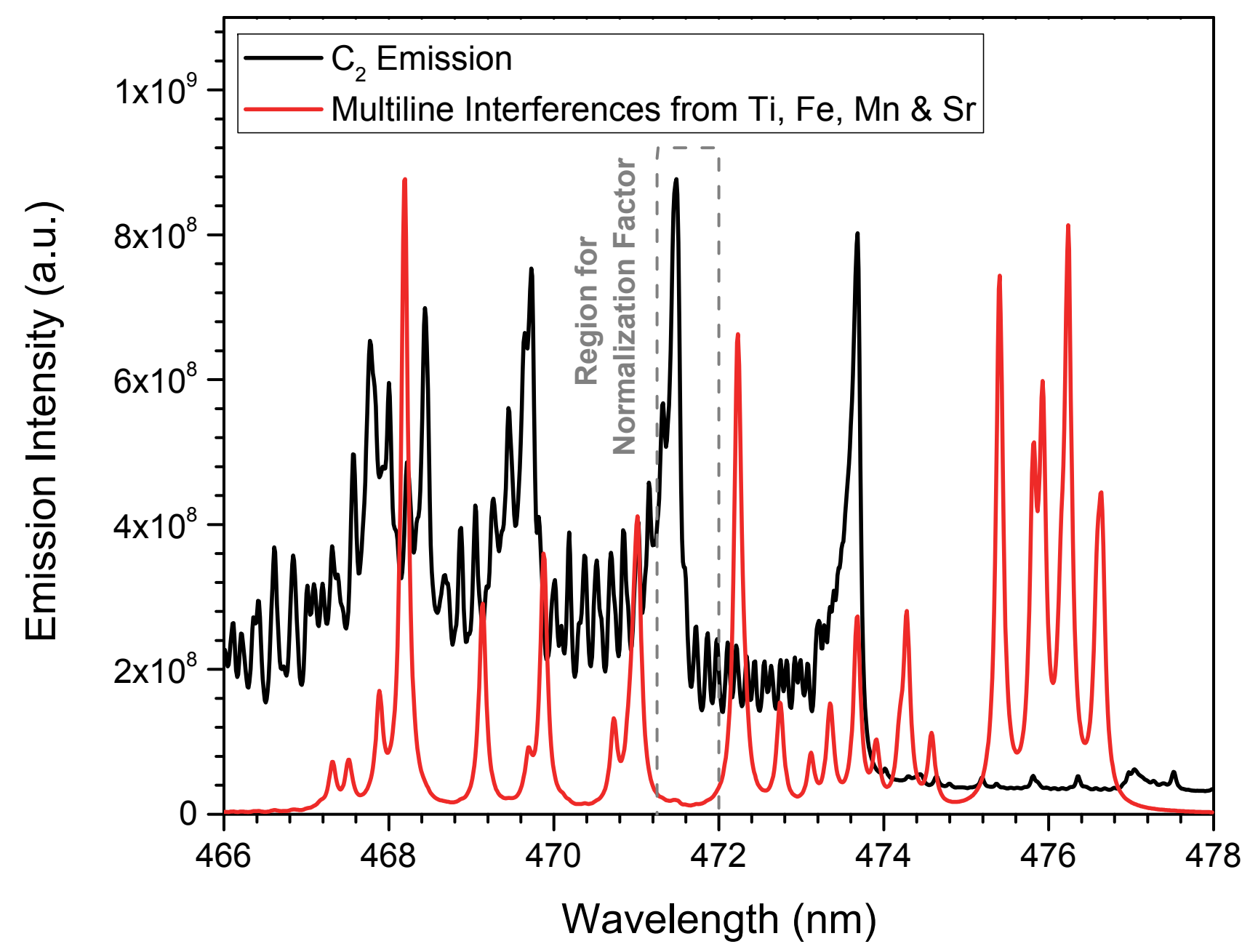


Figure $7 b$

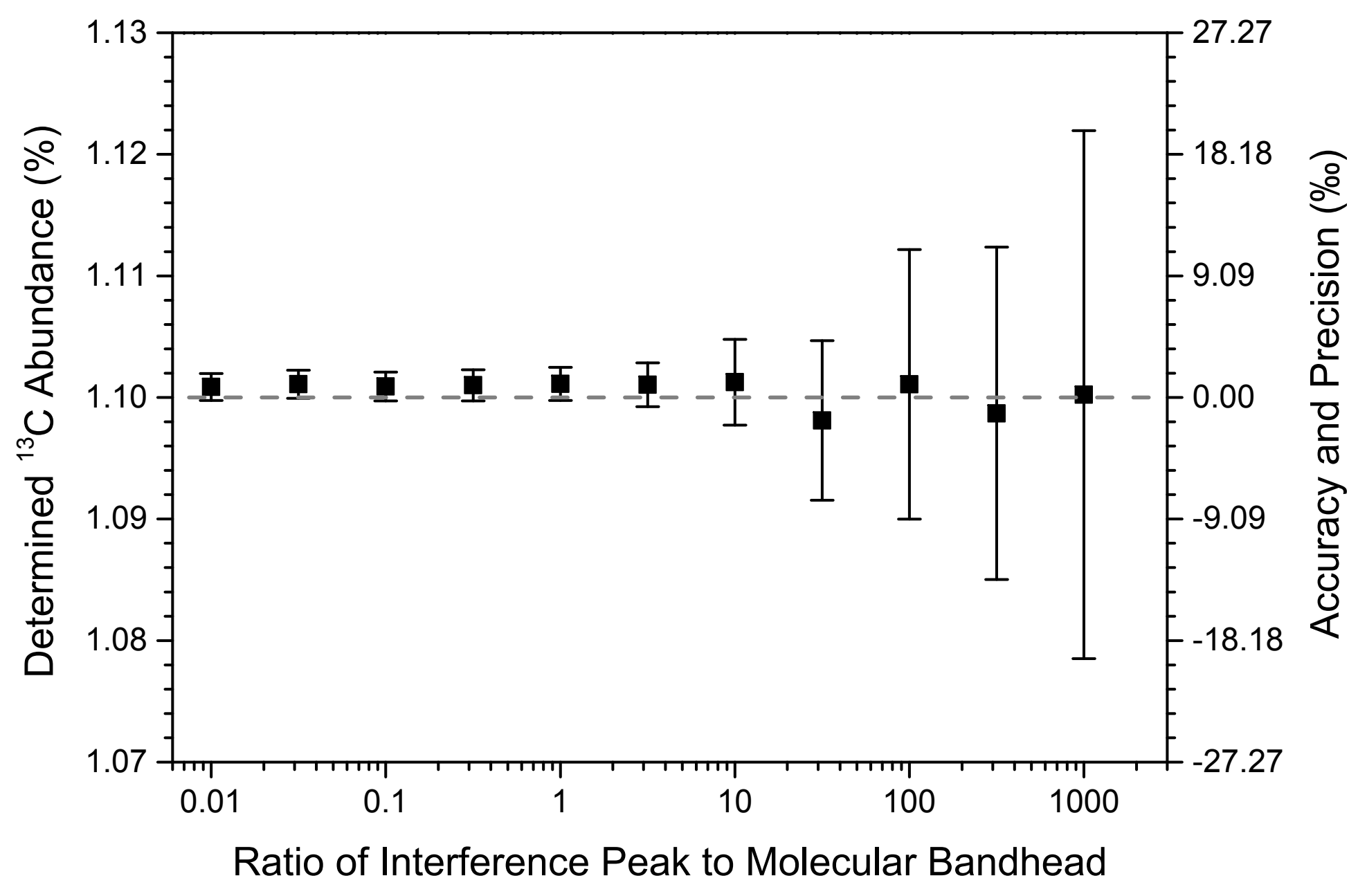


Figure 8a

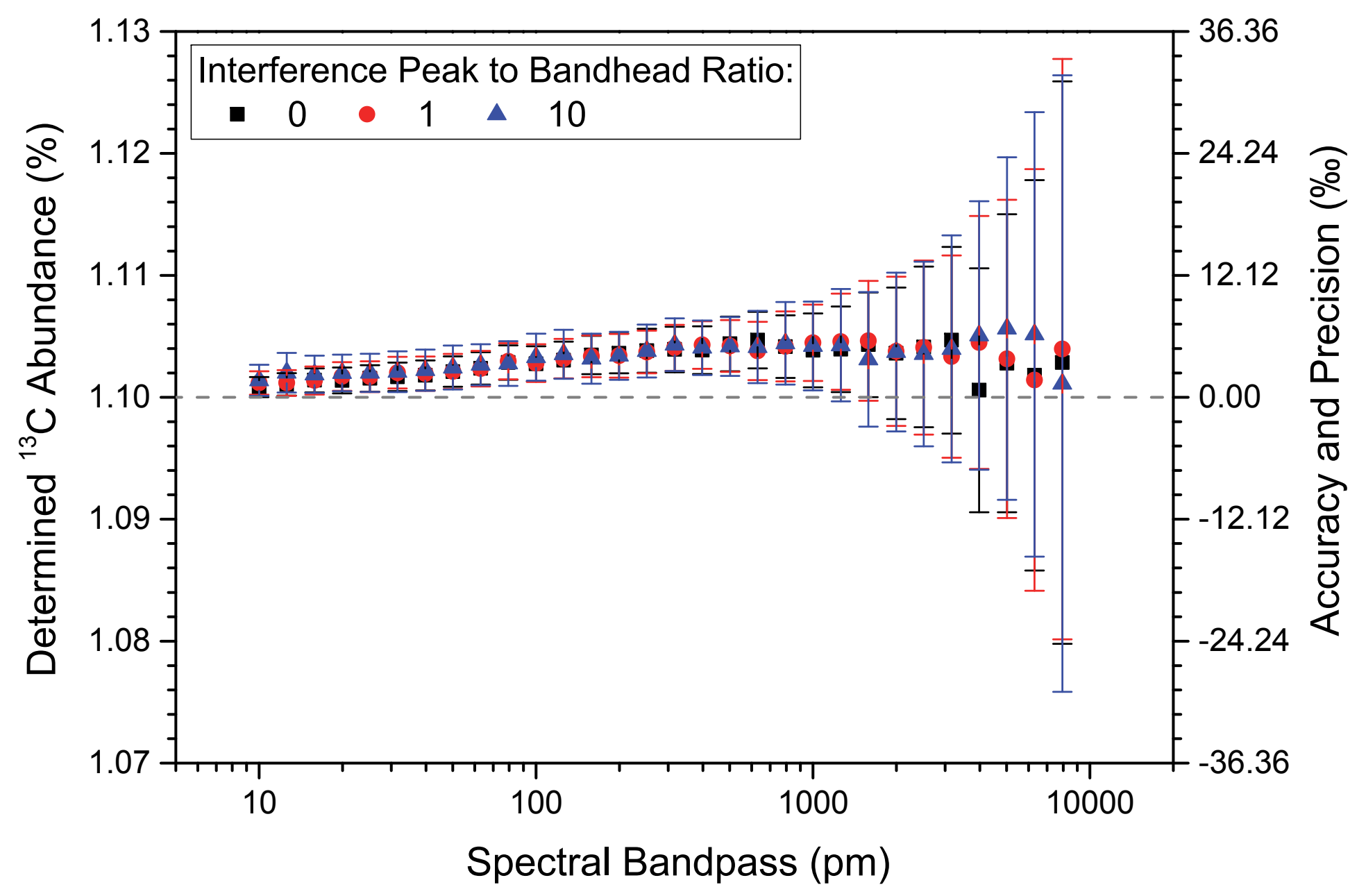


Figure $8 b$

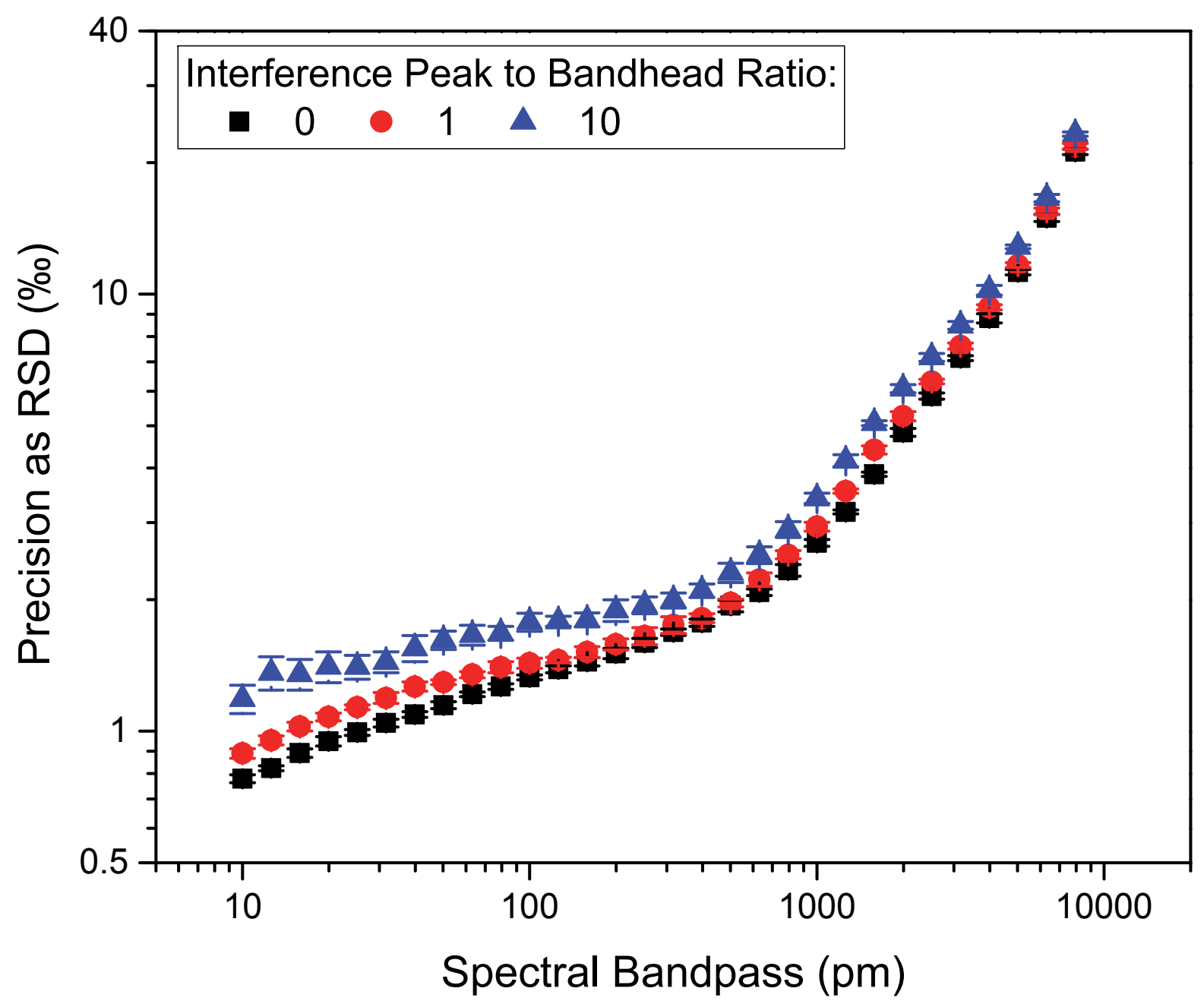

